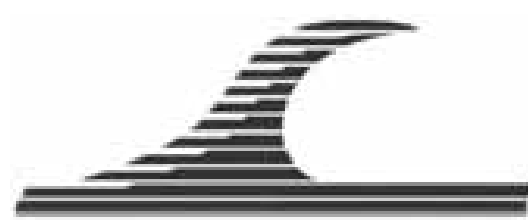

Revue Paralia, Volume 6 (2013) pp 9.1-9.30

Mots-clés : Transport de sédiments en suspension, Distribution verticale de la concentration, Régime non stationnaire, Dépôt, Erosion, Loi de Rouse-Vanoni.

(C) Editions Paralia CFL

\title{
Description non stationnaire de la distribution verticale des sédiments transportés en suspension par les écoulements à surface libre, en présence de dépôt et d'érosion
}

\section{Martin SANCHEZ ${ }^{1}$}

1. Université de Nantes, Faculté des Sciences et des Techniques,

UMR-6112 du CNRS - Planétologie et Géodynamique, 2 rue de la Houssinière, BP 92208, 44322 Nantes cedex 3, France.martin.sanchez@univ-nantes.fr

\section{Résumé :}

Cette étude est basée sur des données issues de la solution numérique d'une équation de transport 1DV qui ont servi à développer un modèle descriptif du profil vertical de la concentration en matières en suspension, en relation avec l'étude du transport de sédiments par les écoulements à surface libre.

Pour un régime sédimentaire non stationnaire sans dépôt et sans érosion, une première description de la répartition verticale de la concentration est proposée. Celle-ci, semblable à l'équation générale du profil de la concentration de la solution stationnaire, inclut un paramètre nommé alpha, dont la valeur tend toujours vers sa valeur théorique terminale correspondant au régime stationnaire.

En présence de dépôt ou d'érosion, si les variables du problème restent constantes et si les échanges solides avec le fond sont proportionnels à la vitesse de chute des sédiments multipliée par la concentration au fond, il est constaté dans cette étude que le profil de la concentration tend vers une forme terminale. Suivant l'approche retenue, la modification de la forme du profil de la concentration due aux échanges avec le fond est décrite par une fonction incluant un deuxième paramètre désigné bêta.

Le modèle complet pour le profil vertical de la concentration en régime non stationnaire avec érosion et dépôt inclut ainsi, deux paramètres adimensionnels, alpha et bêta, et un paramètre dimensionné correspondant à la concentration de référence. Une stationnarité des paramètres alpha et bêta est possible avec érosion ou dépôt, impliquant nécessairement la non stationnarité de la concentration de référence.

Les variations dans le temps des paramètres alpha et bêta sont simulées par deux équations phénoménologiques. Un bon ajustement de l'ensemble des formulations proposées a permis d'aboutir à un modèle stable très fiable qui peut permettre d'améliorer considérablement la précision des modélisations de transport 2DH.

Soumis le 2 octobre 2013, accepté le 28 octobre 2013, en ligne le 6 novembre 2013.

La seule version examinée est celle écrite en français. La ou les autres versions n'étant pas examinées par le comité de rédaction de la revue, sont donc publiées sous l'entière responsabilité du ou des auteurs.

A TRANSLATED VERSION IN ENGLISH IS AVAILABLE ONLINE

Pour citer cet article :

SANCHEZ M. (2013). Description non stationnaire de la distribution verticale des sédiments transportés en suspension par les écoulements à surface libre, en présence de dépôt et d'érosion. Revue Paralia, Vol. 6, pp 9.1-9.30. 


\section{Introduction}

Avec les ordinateurs actuels et pour un coût de calcul relativement faible, les modèles hydrodynamiques 2DH utilisés dans les milieux océaniques, côtiers, lacustres, estuariens et fluviaux, permettent les applications sur des longues durées, à une échelle géographique fine couvrant des grandes étendues.

Lorsque les variables étudiées par ces modèles peuvent être correctement décrites suivant la verticale, les résultats obtenus sont très proches de ceux issus des modèles tridimensionnels. Deux applications réussies suivant cette démarche sont: (i) les modèles de propagation de la houle sur des fonds peu inclinés qui décrivent la distribution verticale du potentiel de vitesses avec la théorie de Stokes-Airy et (ii) les modèles courantologiques de fluides homogènes qui intègrent sur toute la profondeur l'effet des contraintes de cisaillement sur les vitesses moyennes.

L'un des objets de cet article est d'inclure, dans les modèles 2DH de transport de matières en suspension par les écoulements à surface libre, une description précise de la répartition verticale des sédiments. Au-delà de la loi de Rouse-Vanoni qui a été obtenue pour un régime uniforme et stationnaire, le modèle développé peut s'appliquer en régime non stationnaire et non uniforme, quand les échanges solides avec le fond sont paramétrés par un taux de dépôt ou par un taux d'érosion.

Par ce modèle, la concentration de référence au fond peut être connue afin d'évaluer convenablement le taux de dépôt. Ce modèle permet aussi de connaître à tout moment les distributions verticales de la matière solide en suspension, à toutes fins utiles.

\section{Théorie}

Dans les problèmes étudiés dans cet article, les sédiments fins en suspension sont transportés par les courants à la même vitesse horizontale que ceux-ci. Dans un écoulement turbulent 3D, la valeur instantanée de la concentration locale $c$ en matières en suspension fluctue autour d'une valeur moyenne $C$. La décomposition de $c$ s'écrit comme suit :

$c=C+c^{\prime}$

où $c^{\prime}$ est la partie fluctuante de la concentration. Si la diffusion moléculaire des sédiments en suspension est négligée, la concentration $C$ dans un écoulement turbulent est régie par l'équation de transport suivante (référentiel $O x y z$ avec $z$ en coordonnée verticale) :

$\frac{\partial C}{\partial t}+V_{x} \frac{\partial C}{\partial x}+V_{y} \frac{\partial C}{\partial y}+V_{z} \frac{\partial C}{\partial z}=-\frac{\partial\left\langle v_{x}{ }^{\prime} c^{\prime}\right\rangle}{\partial x}-\frac{\partial\left\langle v_{y}{ }^{\prime} c^{\prime}\right\rangle}{\partial y}-\frac{\partial\left\langle v_{z}{ }^{\prime} c^{\prime}\right\rangle}{\partial z}+\frac{\partial(W C)}{\partial z}$

où $W$ est la valeur locale de la vitesse de chute moyenne des matières en suspension, $V_{x}$, $V_{y}$ et $V_{z}$ sont les composantes locales de la vitesse caractérisant l'écoulement turbulent moyen, et les termes $\left\langle v_{x}{ }^{\prime} c^{\prime}\right\rangle,\left\langle v_{y}{ }^{\prime} c^{\prime}\right\rangle,\left\langle v_{z}{ }^{\prime} c^{\prime}\right\rangle$, représentent les corrélations temporelles entre les composantes de la vitesse fluctuante et la concentration fluctuante; ces termes 
Description non stationnaire de la distribution verticale des sédiments transportés en suspension par les écoulements à surface libre, en présence de dépôt et d'érosion : 9.3

traduisent le transfert de masse lié à la turbulence. Usuellement ils sont modélisés à travers les lois de diffusion de Fick-Boussinesq suivantes :

$\left\langle v_{x}{ }^{\prime} c^{\prime}\right\rangle=-K_{x} \frac{\partial C}{\partial x},\left\langle v_{y}{ }^{\prime} c^{\prime}\right\rangle=-K_{y} \frac{\partial C}{\partial y},\left\langle v_{z}{ }^{\prime} c^{\prime}\right\rangle=-K_{z} \frac{\partial C}{\partial z}$

où $K_{x}, K_{y}$ et $K_{z}$ sont les coefficients de diffusion turbulente de masse selon les directions $O x, O y$ et $O z$ respectivement. L'équation de transport $3 \mathrm{D}$ devient :

$\frac{\partial C}{\partial t}+V_{x} \frac{\partial C}{\partial x}+V_{y} \frac{\partial C}{\partial y}+V_{z} \frac{\partial C}{\partial z}=\frac{\partial}{\partial x}\left(K_{x} \frac{\partial C}{\partial x}\right)+\frac{\partial}{\partial y}\left(K_{y} \frac{\partial C}{\partial y}\right)+\frac{\partial}{\partial z}\left(K_{z} \frac{\partial C}{\partial z}\right)+\frac{\partial(W C)}{\partial z}$

La solution de cette équation doit satisfaire à une condition de frontière relative aux échanges de sédiments entre l'écoulement et le fond.

Si le fond se situe en $z=0$, en cas de remise en suspension décrite par un taux d'érosion effectif $E^{e f}$ (terme source en $\mathrm{kg} \mathrm{m}^{-2} \mathrm{~s}^{-1}$ ), la condition de frontière est :

$\left.\left(-W C-K_{z} \frac{\partial C}{\partial z}\right)\right|_{z=0}=E^{e f}$

En cas de dépôt la condition de frontière est :

$\left.\left(-W C-K_{z} \frac{\partial C}{\partial z}\right)\right|_{z=0}=-D^{e f}$

où $D^{e f}$ représente le taux de dépôt effectif (terme puits en $\mathrm{kg} \mathrm{m}^{-2} \mathrm{~s}^{-1}$ ) qui peut être décrit par la formulation suivante (KRONE, 1986 ; METHA, 1986) :

$D^{e f}=p W_{0} C_{0}$

où d'une part $C_{0}$ est la concentration en matières en suspension au fond de l'écoulement et $W_{0}$ la vitesse de chute moyenne de ces matières. D'autre part le terme $p$ représente selon KRONE (1986), la probabilité $[0 ; 1]$ que les sédiments touchant le fond adhèrent à celui-ci. Si $p=0$ toutes les particules touchant le fond sont immédiatement remises en suspension par l'écoulement et le taux de dépôt effectif est nul.

Si le régime sédimentaire est uniforme et stationnaire, le problème est considérablement simplifié et la distribution verticale de $C$ obéit à :

$W C=-K_{z} \frac{\partial C}{\partial z}$

L'intégration de cette équation permet d'obtenir la loi de Rouse-Vanoni généralisée pour la distribution verticale des matières en suspension en régime stationnaire (ORTON \& KINEKE, 2001 ; SANCHEZ et al., 2005) :

$\left.C(z)\right|_{\text {stat }}=C_{0} \exp \left(-\int_{0}^{z} \frac{W}{K_{z}} \mathrm{~d} z\right)$

L'équation précédente peut admettre une solution analytique dépendant des expressions de $K_{z}$ et de $W$. Quelques solutions connues sont présentées dans l'appendice 1 à la fin de ce manuscrit. Dans ce qui suit, ces deux grandeurs sont considérées être indépendantes de $z$. L'expression 9 de la concentration devient alors : 


$$
\left.C\left(z^{\circ}\right)\right|_{\text {stat }}=C_{0} \exp \left(-P^{e} z^{\circ}\right)
$$

où $z^{\circ}=z / d$ est la coordonnée verticale adimensionnelle, $d$ la profondeur et $P^{e}$ le nombre de Peclet caractéristique des transferts verticaux de sédiments par convection-diffusion : $P^{e}=\frac{W d}{K_{z}}$

Une relation usuelle pour évaluer $K_{z}$ est la suivante :

$$
K_{z}=\frac{\kappa}{6} U_{c} d
$$

où $U_{c}$ est la vitesse de cisaillement et $\kappa=0,4$ la constante universelle de Karman.

Par l'intégration de l'équation 10 on obtient l'expression du rapport $C_{0} / \bar{C}$ (où $\bar{C}$ est la concentration moyenne sur la verticale) :

$$
\left.\frac{C_{0}}{\bar{C}}\right|_{\text {stat }}=\frac{P^{e}}{1-\exp \left(-P^{e}\right)}
$$

L'équation 13 pourrait être utilisée dans les modélisations 2DH pour estimer la concentration au fond $C_{0}$ en fonction de la concentration moyenne sur la verticale $\bar{C}$. En effet la connaissance de $C_{0}$ est nécessaire afin d'estimer de façon fiable le taux de dépôt par l'équation 7.

Cependant, selon les modélisations hydrosédimentaires et en accord avec les travaux de simulations numériques menés par TEETER (1986), dès que la sédimentation des matières en suspension commence à se produire, le rapport $C_{0} / \bar{C}$ diminue par rapport à la valeur donnée par l'équation 13. Cette diminution s'explique par la réduction de la concentration qui est relativement plus importante près du fond comme indiqué sur la figure 1. Une formulation de TEETER (1986) qui permet de calculer le rapport $C_{0} / \bar{C}$ dans les modèles actuels (LUMBORG \& WINDELIN, 2003) s'écrit comme suit :

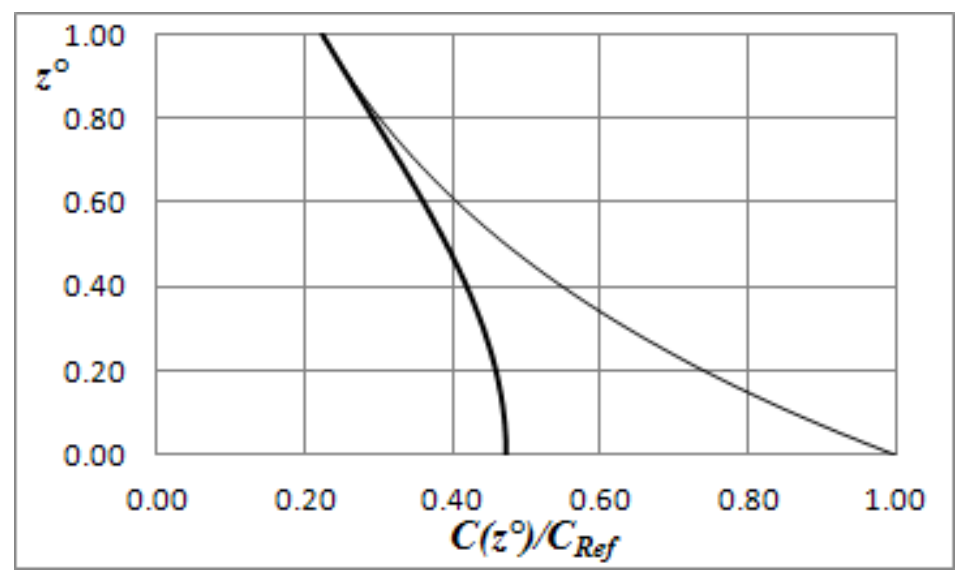

Figure 1. Illustration de la modification du profil vertical de la concentration provoquée par la sédimentation. En trait fin : loi stationnaire. En trait épais : profil observé pendant une période de dépôt $\left(z^{\circ}=0=\right.$ fond $; z^{\circ}=1=$ surface). 
Description non stationnaire de la distribution verticale des sédiments transportés en suspension par les écoulements à surface libre, en présence de dépôt et d'érosion : 9.5

$$
\frac{C_{0}}{\bar{C}} \approx 1+\frac{P^{e}}{1,25+4,75 p^{2,5}}
$$

Des études sur le transport de sédiments non-cohésifs montrent que lorsque le nombre de Peclet dépasse un seuil critique $P_{c r}^{e}$ dont la valeur est comprise entre 9 et 15 le transport en suspension ne peut plus se produire (TEETER, 1986). Ce critère doit pouvoir s'appliquer aussi aux sédiments fins quand la valeur du nombre de Peclet reste inférieure à cette valeur critique assez longtemps par rapport à la durée nécessaire pour que les particules en suspension se déposent.

\section{Méthodes}

Afin de comprendre le comportement dynamique des sédiments dans la colonne d'eau, un modèle numérique $1 \mathrm{DV}$ a été mis au point. Celui-ci est régi par l'équation suivante :

$$
\frac{\partial C}{\partial t}=\frac{\partial}{\partial z}\left(\frac{\kappa}{6} U_{c} d \frac{\partial C}{\partial z}+W C\right)
$$

Des tests ont été effectués avec une large gamme de valeurs des variables du problème couvrant la plupart des cas réels de transport des sédiments fins par les écoulements côtiers et estuariens. Lors de ces tests on a combiné différentes valeurs pour les variables. Parmi les valeurs étudiées on peut citer :

- 8 valeurs de $d$ (en $\mathrm{m}): 0,25 ; 0,5 ; 1 ; 2 ; 4 ; 8 ; 16$ et 32 .

- 6 valeurs de $W\left(\mathrm{en} \mathrm{mm} \mathrm{s}^{-1}\right): 0,05 ; 0,1 ; 0,2 ; 0,4 ; 0,8$ et 1,6 .

- Des valeurs de $U_{c}\left(\mathrm{en} \mathrm{m} \mathrm{s}^{-1}\right)$ variables comprises entre 0,00002 et 0,3 .

Le modèle numérique décompose la colonne d'eau en $N$ couches d'épaisseur adimensionnelle constante égale à $\Delta z^{\circ}=\Delta z / d=1 / N$. Dans la plupart des tests $N$ a été fixé à 50 , mais dans des certains tests spécifiques on a utilisé $N=100$. A chaque instant $t$ de la modélisation, on connaît la concentration $C_{i}$ au milieu de chaque couche $i$ de la colonne d'eau dont la cote adimensionnelle par rapport au fond est $z_{i}^{\circ}=(i-1 / 2) \Delta z^{\circ}$.

\subsection{Régime non stationnaire sans érosion et sans dépôt}

Les premiers tests ont été effectués avec une vitesse de cisaillement cycliquement variable donnée par l'expression suivante :

$U_{c}=U_{c-\text { moy }}[1+A m p l \times \cos (\omega t)]$

où $U_{c-m o y}$ est la vitesse de cisaillement moyenne du test, $\mathrm{Ampl}$ un paramètre définissant l'importance des oscillations de la valeur de $U_{c}$ et $\omega=2 \pi / T$ la pulsation qui est reliée à la période $T$ des oscillations.

Pour la plupart des tests, le paramètre $\mathrm{Ampl}$ a été fixé à 0,80 (sauf dans certains cas où sa valeur est indiquée) et la période $T$ à $22320 \mathrm{~s}$, ce qui est la moitié de la valeur théorique de la période de la marée semi-diurne. Pour le paramètre $U_{c-\text { moy }}\left(\mathrm{en}^{-1}\right)$ les 4 valeurs suivantes ont notamment été étudiées : 0,$01 ; 0,02 ; 0,04$ et 0,08 . 


\subsubsection{Description du profil vertical de la concentration sans dépôt et sans érosion}

Les premiers tests ont montré que le profil vertical de la concentration restait, dans la plupart des cas, proche d'une loi exponentielle décroissante. Il faut néanmoins indiquer que ces profils ne sont pas exactement en conformité avec cette loi. Afin d'ajuster à chaque instant $t$ de la modélisation, une loi exponentielle approchant le profil de concentrations, on a utilisé la méthode des moindres carrés.

Selon cette méthode, si une variable $Y$ est reliée linéairement à une variable $X$, et que $\hat{Y}=A+B X$ représente la meilleure approche de $Y$ ajustée en conformité avec ce critère, alors les paramètres $A$ et $B$ sont donnés par :

$$
\begin{gathered}
A=\frac{N \sum_{i=1}^{N} X_{i} Y_{i}-\sum_{i=1}^{N} X_{i} \sum_{i=1}^{N} Y_{i}}{N \sum_{i=1}^{N} X_{i}^{2}-\left(\sum_{i=1}^{N} X_{i}\right)^{2}} \\
B=\frac{\sum_{i=1}^{N} X_{i}^{2} \sum_{i=1}^{N} Y_{i}-\sum_{i=1}^{N} X_{i} Y_{i} \sum_{i=1}^{N} X_{i}}{N \sum_{i=1}^{N} X_{i}^{2}-\left(\sum_{i=1}^{N} X_{i}\right)^{2}}
\end{gathered}
$$

où $X_{i}$ et $Y_{i}$ sont les couples associés des valeurs expérimentales de ces variables et $N$ le nombre total de ces couples.

Si le profil vertical de la concentration est approché par la loi suivante :

$$
\hat{C}\left(z^{\circ}\right)=\hat{c} \exp \left(-\hat{\alpha} z^{\circ}\right)
$$

où $\hat{c}$ est une concentration de référence et $\hat{\alpha}$ le paramètre de la loi, alors, le problème est linéarisé en procédant au changement de variables qui suit : $X_{i}=z^{\circ}{ }_{i}$ et $Y_{i}=\ln \left(C_{i}\right)$. Dans ce cas les paramètres de la loi sont donnés par : $\hat{\alpha}=-A$ et $\hat{c}=\exp (B)$.

\subsubsection{Caractérisation de l'erreur entre la loi exponentielle ajustée et le profil vertical de} la concentration selon le modèle $1 \mathrm{DV}$

L'erreur relative liée à l'estimation de $C_{i}$ par $\hat{C}$, est définie comme suit :

$$
\hat{e}\left(z^{\circ}\right)=\frac{\hat{C}\left(z^{\circ}\right)-C_{i}\left(z^{\circ}\right)}{C_{i}\left(z^{\circ}\right)}
$$

A chaque instant de la modélisation, la fonction $\hat{e}\left(z^{\circ}\right)$ peut être évaluée à partir des valeurs $C_{i}\left(z^{\circ}\right)$ issues du modèle numérique $1 \mathrm{DV}$ et des valeurs ajustées des paramètres $\hat{c}$ et $\hat{\alpha}$.

\subsection{Régime non stationnaire avec prise en compte du dépôt et de l'érosion}

Des tests du modèle numérique 1DV avec dépôt et/ou érosion ont été effectués en combinant les différentes valeurs des grandeurs du problème données précédemment. 
Description non stationnaire de la distribution verticale des sédiments transportés en suspension par les écoulements à surface libre, en présence de dépôt et d'érosion : 9.7

Les tests de dépôt ont été effectués avec les valeurs suivantes du paramètre $p$ de la loi de Krone (équation 7$)$ : 0,$125 ; 0,25 ; 0,5$ et 1 .

Pour caractériser les tests de remise en suspension des sédiments, on a défini un taux d'érosion adimensionnel $q$ comme suit :

$q=\frac{E^{e f}}{W_{0} C_{0}}$

Les valeurs étudiées du paramètre $q$ sont $: 0,125 ; 0,25 ; 0,5 ; 1 ; 2 ; 4 ; 8$ et 16 .

Un nombre de tests réduit a été effectué en fixant la valeur du rapport $E^{e f} /\left(W_{\text {init }} \times C_{\text {init }}\right)$, où $W_{\text {init }}$ et $C_{\text {init }}$ désignent les valeurs initiales (à $t=0$ ) des variables $W$ et $C$ respectivement.

\section{Résultats des simulations numériques $1 \mathrm{DV}$ et développement d'un modèle phénoménologique}

\subsection{Transport sédimentaire sans dépôt et sans érosion}

\subsubsection{Erreur relative locale liée à la loi exponentielle ajustée pour décrire le profil} vertical de la concentration

Les tests examinés correspondent à une variation de $U_{c}$ suivant l'équation 16 , avec des valeurs variables des grandeurs $U_{c-m o y}, W$ et $d$.

L'étude de la fonction $\hat{e}\left(z^{\circ}\right)$ caractérisant l'erreur relative locale liée à l'approche de la valeur de $C_{i}$ obtenue avec le modèle théorique $1 \mathrm{DV}$, par l'équation de $\hat{C}$ selon la loi ajustée, montre que dans tous les cas les profils de $\hat{e}\left(z^{\circ}\right)$ restent similaires (figure 2). Le maximum de $|\hat{e}|$ est toujours observé au fond $\left(\right.$ en $\left.z^{\circ}=0\right)$. Le signe de $\hat{e}$ à la surface est toujours contraire à celui correspondant au fond. Le profil vertical de l'erreur relative comporte systématiquement 3 passages par $\hat{e}=0$. Bien que les lieux $z^{\circ}$ de ces passages varient légèrement d'un cas à un autre, on peut avancer les valeurs suivantes :

- Passage supérieur: $\quad z^{\circ} \approx 0,90$

- Passage intermédiaire : $z^{\circ} \approx 0,51$

- Passage inférieur: $\quad z^{\circ} \approx 0,12$

L'étude de l'erreur relative a permis de dresser la figure 3 avec 51840 valeurs de $\hat{e}\left(z^{\circ}=0\right)$ correspondant à la combinaison des différentes valeurs des grandeurs du problème.

Dans $90 \%$ des cas étudiés la valeur absolue de $\hat{e}\left(z^{\circ}=0\right)$ reste inférieure à 0,03 et dans $99 \%$ des cas inférieure à 0,23 . Cependant, dans certains cas les valeurs maximales de $\left|\hat{e}\left(z^{\circ}=0\right)\right|$ ont dépassé une valeur de 0,40 . Il a été constaté que ces erreurs maximales correspondent aux plus grandes valeurs du nombre de Peclet qui atteignent dans les modélisations une valeur de 12. Il faut signaler que ces valeurs du nombre de Peclet, dépassant le seuil critique $P_{c r}^{e}$ au-dessus duquel le transport en suspension ne peut plus se produire, impliquent un dépôt des sédiments qui n'est pas permis dans cette première série de modélisations. 
Une relation très étroite a été trouvée entre les valeurs instantanées de $\hat{e}\left(z^{\circ}=0\right)$ et quelques grandeurs caractéristiques du problème (voir figure 3 ). La loi suivante a notamment été ajustée (celle-ci surestime l'erreur pour les grandes valeurs de $d \hat{\alpha} / d t$ ) :

$\hat{e}(0)_{\text {estim }} \approx-0,108 \frac{d}{U_{c}} \frac{d \hat{\alpha}}{d t}$
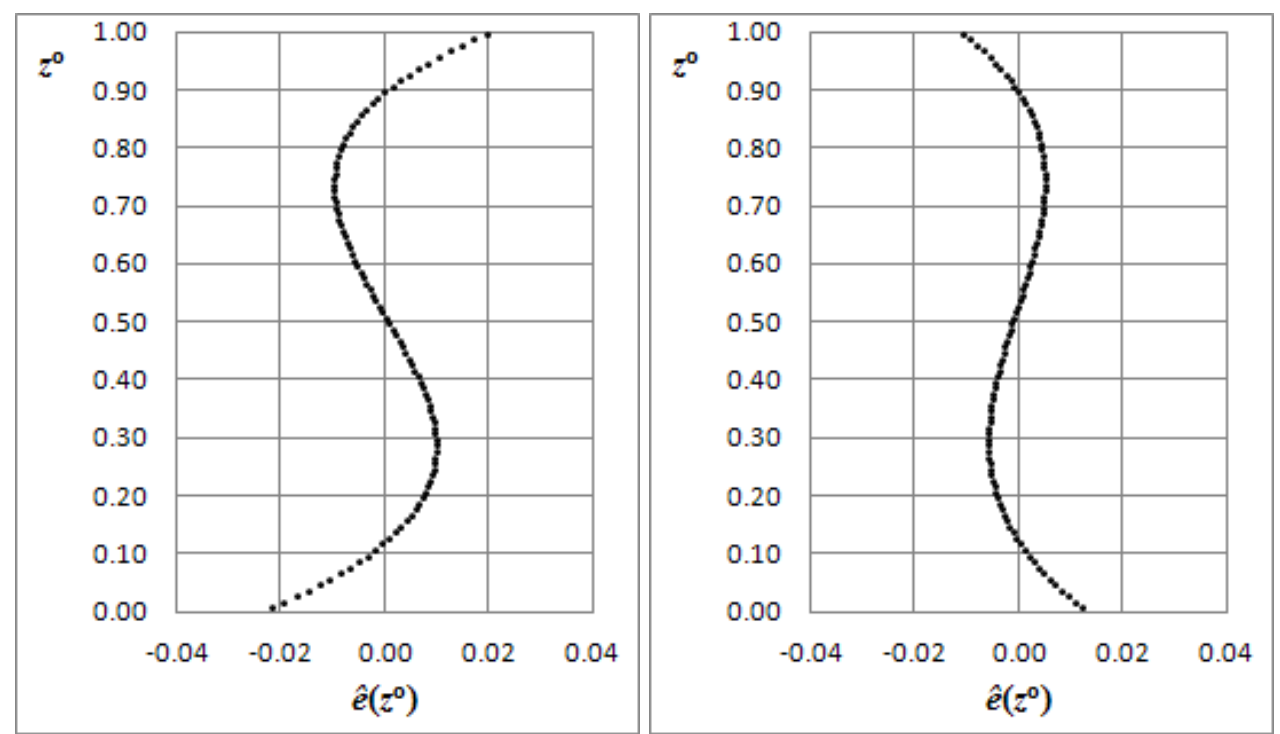

Figure 2. Exemples de deux profils verticaux de $\hat{e}\left(z^{\circ}\right)$ correspondant aux erreurs maximales constatées pour le cas test défini par : $U_{c-m o y}=0,02 \mathrm{~m} \mathrm{~s}^{-1} ; W=0,0002 \mathrm{~m} \mathrm{~s}^{-1}$ et $d=8 \mathrm{~m}$.

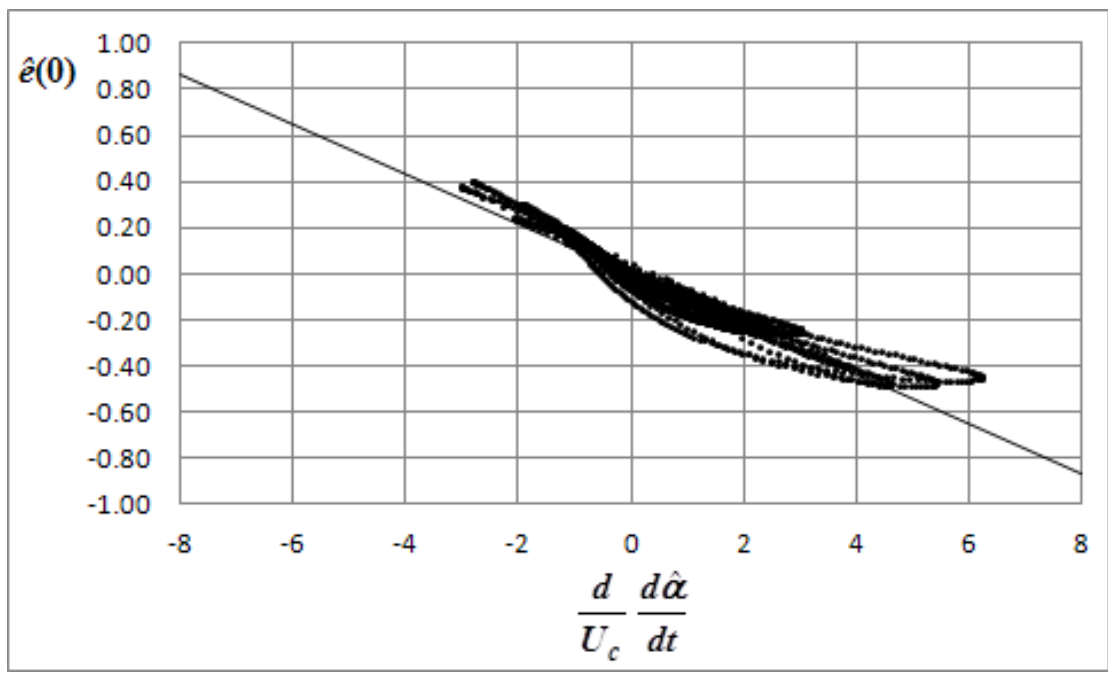

Figure 3. Relation entre $\hat{e}\left(z^{\circ}=0\right)$ et $\left(d / U_{c}\right) \times d \hat{\alpha} / d t$ selon les résultats des modélisations numériques $1 \mathrm{DV}$ (la figure comporte 51840 points expérimentaux).

En raison du fort coefficient de corrélation justifiant l'équation 22, celle-ci pourrait être incluse dans un modèle pour améliorer sa précision. Cependant, sachant que les valeurs 
Description non stationnaire de la distribution verticale des sédiments transportés en suspension par les écoulements à surface libre, en présence de dépôt et d'érosion : 9.9

extrêmes de $\hat{e}\left(z^{\circ}=0\right)$ sont observées pour des conditions de dépôt bien marqué, cette possibilité n'est pas retenue car l'étude du transport sédimentaire sans dépôt et sans érosion ne comporte que peu d'intérêt.

On doit noter qu'une valeur positive de $d \hat{\alpha} / d t$, qui correspond à une période d'accroissement de la concentration au fond au détriment de celle en surface, s'accompagne d'une valeur négative de $\hat{e}(0)$, laquelle indique qu'au fond, la valeur de $\hat{C}$ selon la loi ajustée, est inférieure à la concentration $C_{i}$ évaluée avec le modèle 1DV.

\subsubsection{Modélisation du profil vertical de la concentration en régime non stationnaire sans érosion et sans dépôt}

Après avoir vérifié, qu'en similitude avec les équations 10 et 19 , le rapport $C / C_{0}$ peut être modélisé correctement par une fonction $F\left(z^{\circ} ; \alpha\right)=\exp \left(-\alpha z^{\circ}\right)$, un modèle phénoménologique est utilisé pour décrire le profil vertical de la concentration en régime non stationnaire sans dépôt et sans érosion. Celui-ci s'écrit comme suit :

$\widetilde{C}\left(z^{\circ}\right)=\widetilde{c} \exp \left(-\tilde{\alpha} z^{\circ}\right)$

où $\widetilde{c}$ est la concentration de référence au fond et $\tilde{\alpha}$ le paramètre de la loi (plus la valeur du paramètre $\widetilde{\alpha}$ est petite, plus la distribution des sédiments sur la verticale est uniforme).

Les tracés de $\hat{\alpha}$ et de $P^{e}$ en fonction du temps montrent que dans tous les cas étudiés le signal $\hat{\alpha}(t)$ est en retard de phase sur $P^{e}(t)$. A tout instant $t$, la valeur de $\hat{\alpha}$ tend vers la valeur de $P^{e}$ (figure 4 ). On constate aussi que le maximum et le minimum du signal de $\hat{\alpha}$ correspond à l'instant où les deux signaux se croisent. Enfin, l'analyse des résultats montre que le déphasage dépend essentiellement du rapport $U_{c} / d$. Sur la base de ces observations le modèle retenu pour simuler la variation dans le temps de $\tilde{\alpha}$ est le suivant :

$\frac{d \widetilde{\alpha}}{d t}=c_{\alpha} \frac{U_{c}}{d}\left(\alpha_{\infty}-\tilde{\alpha}\right)$

où $\alpha_{\infty}=P^{e}$ est la valeur terminale du paramètre $\tilde{\alpha}$ en régime stationnaire et $c_{\alpha}$ un coefficient du modèle. L'ensemble des résultats numériques du modèle $1 \mathrm{DV}$ ont servi à valider l'équation ci-dessus et à évaluer $c_{\alpha} \approx 0,667$.

Une expression équivalente à l'équation 23 a été utilisée par BELINSKY et al. (2005) pour étudier le chargement d'une colonne d'eau de profondeur $d \rightarrow \infty$ provoqué par une érosion du fond. En admettant l'existence d'une valeur terminale de saturation pour $C_{0}$ ils aboutissent à un modèle quelque peu différent de celui défini par l'équation 24.

La figure 4 est un exemple de résultats obtenus pour un cas test. Cette figure montre les variations temporelles : (i) de $U_{c}$ (en $\mathrm{dm} \mathrm{s}^{-1}$ ), (ii) de $P^{e}$, (iii) du paramètre $\hat{\alpha}$ évalué à partir des résultats obtenus du modèle 1DV par l'ajustement conforme au critère de moindres carrés, et (iv) du paramètre $\widetilde{\alpha}$ obtenu à partir de l'équation 24 résolue numériquement avec la méthode predictor-corrector de Runge-Kutta. En complément, 
cette figure montre $\hat{e}\left(z^{\circ}=0\right)$ évalué expérimentalement d'une part et estimé avec la relation empirique définie par l'équation 22 d'autre part.

Les déphasages les plus importants entre les signaux $P^{e}(t)$ et $\hat{\alpha}(t)$ ont été observés pour $d=32$ m et $U_{c-\text { moy }}=0,01 \mathrm{~m} \mathrm{~s}^{-1}$ qui sont les valeurs étudiées extrêmes opposées de ces deux grandeurs. Les courbes de la figure 5 correspondent à ce cas. En raison de la forte valeur du rapport $d / U_{c}$ les valeurs maximales constatées de $\hat{e}\left(z^{\circ}=0\right)$ sont ici très élevées et coïncident toujours avec les valeurs extrêmes de $P^{e}$.

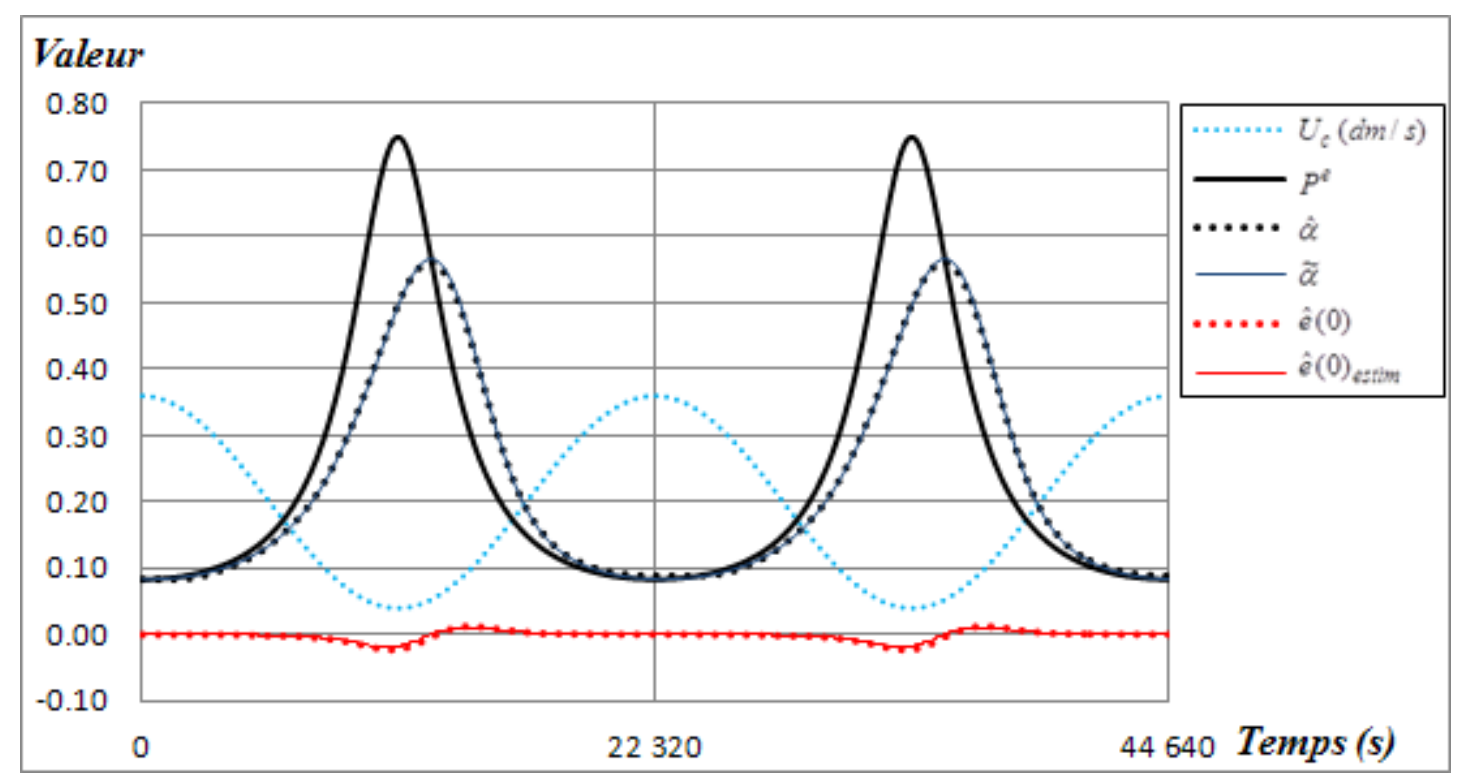

Figure 4. Résultats de validation du modèle phénoménologique en régime non stationnaire, sans érosion et sans dépôt, correspondant au cas test défini par :

$$
U_{c-m o y}=0,02 \mathrm{~m} \mathrm{~s}^{-1} ; W=0,0002 \mathrm{~m} \mathrm{~s}^{-1} \text { et } d=8 \mathrm{~m} \text {. }
$$

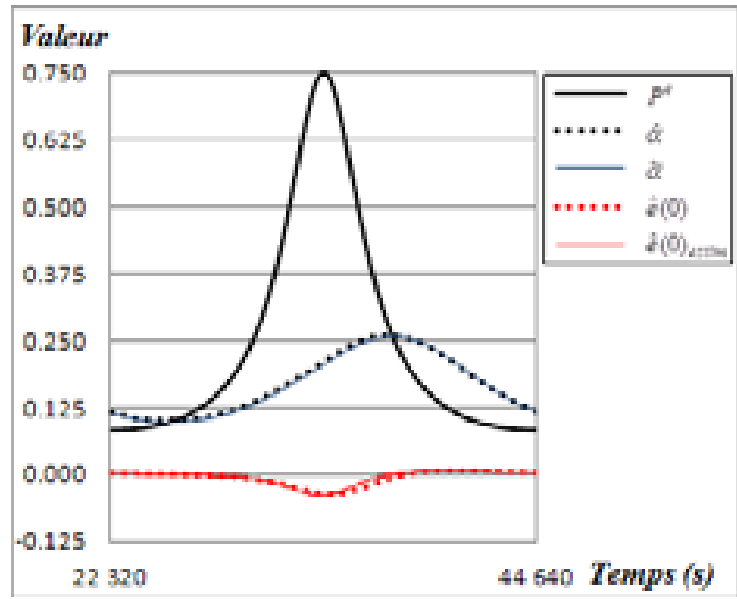

a) $W=0,0001 \mathrm{~m} \mathrm{~s}^{-1}$

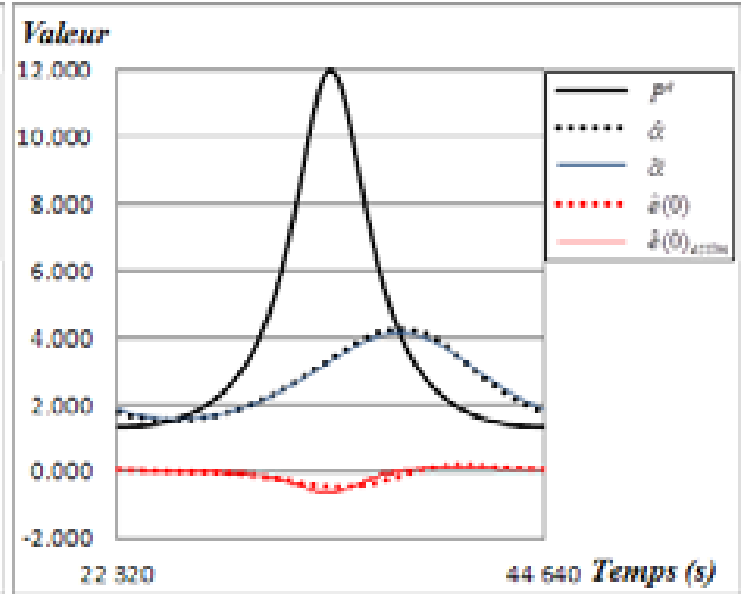

b) $W=0,0016 \mathrm{~m} \mathrm{~s}^{-1}$

Figure 5. Résultats en régime non stationnaire, sans érosion et sans dépôt, correspondant à deux cas tests définis par : $U_{c-m o y}=0,01 \mathrm{~m} \mathrm{~s}^{-1}$ et $d=32 \mathrm{~m}$. 
Description non stationnaire de la distribution verticale des sédiments transportés en suspension par les écoulements à surface libre, en présence de dépôt et d'érosion : 9.11

La figure $5 \mathrm{a}$ correspond à une vitesse de chute $W=0,0001 \mathrm{~m} \mathrm{~s}^{-1}$ et la figure $5 \mathrm{~b}$ à $W=0,0016 \mathrm{~m} \mathrm{~s}^{-1}$. Entre ces deux cas les rapports $\hat{\alpha}(t) / P^{e}$ restent pratiquement identiques avec néanmoins une petite différence impliquant une légère dépendance du coefficient $c_{\alpha}$ du modèle phénoménologique vis-à-vis de la valeur de $\hat{\alpha}$ lorsque celle-ci devient élevée, du même ordre de grandeur que $P_{c r}^{e}$. Cette dépendance est négligée dans cette étude dans la gamme des valeurs examinées pour les variables du problème.

Pour des valeurs du rapport $U_{c-m o y} / d$ supérieures à environ 0,02 le déphasage entre les signaux $P^{e}(t)$ et $\hat{\alpha}(t)$ devient négligeable sauf au voisinage temporel du maximum du nombre de Peclet (figure 6).

De fait, dans un environnement côtier le maximum du nombre de Peclet est observé pendant les renverses de courant quand $U_{c}$ peut tendre vers zéro. Ainsi, au cours d'un cycle de marée le régime sédimentaire peut être considéré stationnaire pendant une partie du temps et non stationnaire pendant la partie complémentaire de ce cycle. Dans la mesure où le signal $U_{c}$ imposé dans les modélisations a une période $T=22320 \mathrm{~s}$ (ce qui est représentatif des ondes de marée semi-diurnes dont la période est de $44640 \mathrm{~s}$ ), on peut avancer le critère suivant pour déterminer si le régime sédimentaire dans un environnement donné est stationnaire ou non stationnaire à un instant donné :

Si $: \frac{U_{c} \times T}{d}>\sim 500$, le régime sédimentaire est quasi-stationnaire

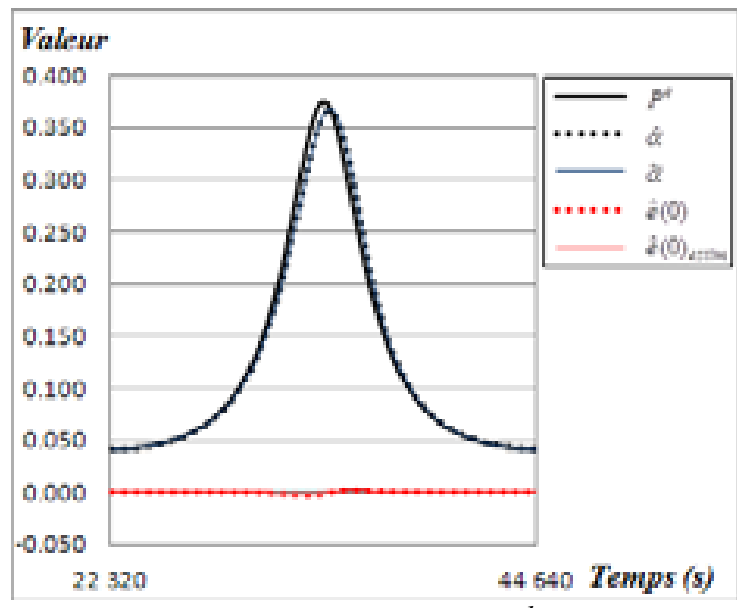

a) $W=0,0001 \mathrm{~m} \mathrm{~s}^{-1}$

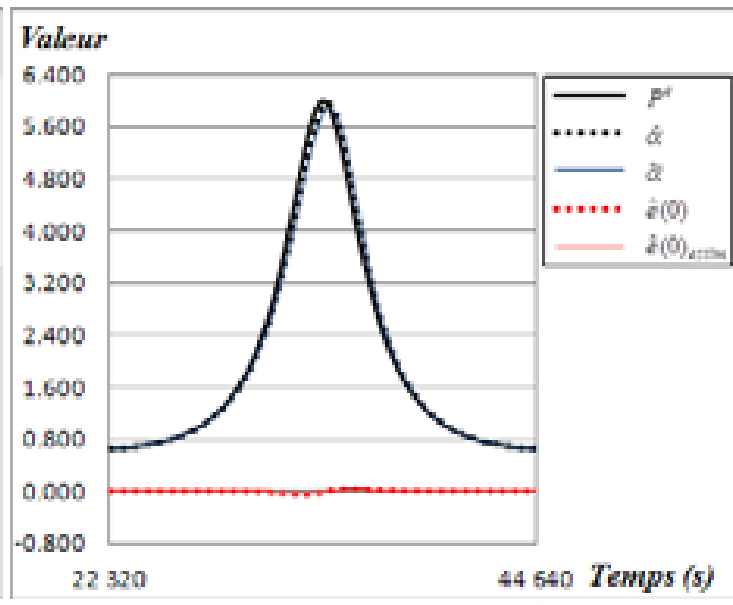

b) $W=0,0016 \mathrm{~m} \mathrm{~s}^{-1}$

Figure 6. Résultats en régime non stationnaire, sans érosion et sans dépôt, correspondant à deux cas tests définis par : $U_{c-m o y}=0,02 \mathrm{~m} \mathrm{~s}^{-1}$ et d $=1 \mathrm{~m}$.

Afin d'explorer la robustesse du modèle phénoménologique, des tests ont été effectués incluant des valeurs $U_{c} \rightarrow 0$ pour lesquels $P^{e} \rightarrow \infty$. Ces tests correspondent à une vitesse de chute $W=0,0002 \mathrm{~m} \mathrm{~s}^{-1}$ et à une variation de la vitesse de cisaillement contrôlée par $U_{c-m o y}=0,02 \mathrm{~m} \mathrm{~s}^{-1}$ et $\mathrm{Ampl}=0,999$, de sorte qu'au cours de la modélisation la valeur 
minimale de $U_{c}$ est de $2 \times 10^{-5} \mathrm{~m} \mathrm{~s}^{-1}$ et la valeur maximale associée du nombre de Peclet vaut 150 . Les résultats correspondants, présentés sur la figure 7 , montrent la validité du modèle phénoménologique dans ce cas extrême. Ces résultats confirment également la dépendance du coefficient $c_{\alpha}$ du modèle phénoménologique vis-à-vis de la valeur de $\hat{\alpha}$. Il semble en effet que pour les fortes valeurs de $\hat{\alpha}$, la valeur du coefficient $c_{\alpha}$ augmente légèrement. Cette dépendance est négligée dans cette étude.

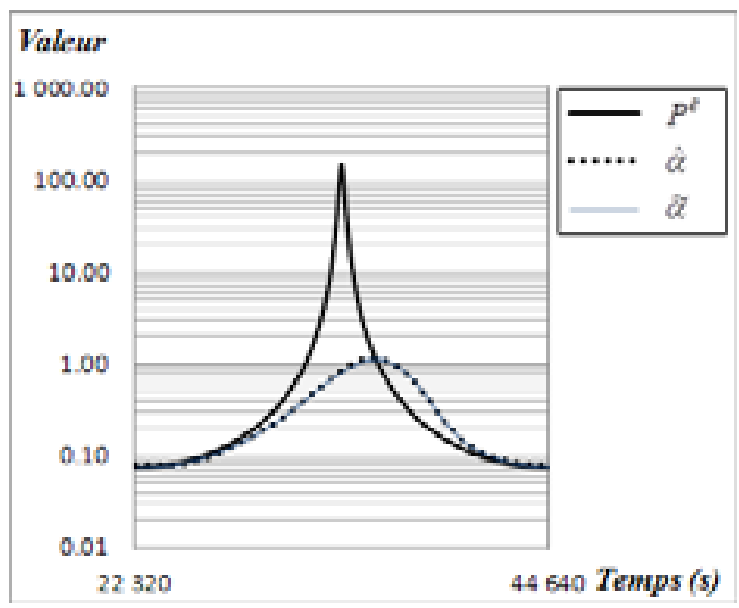

a) $d=8 \mathrm{~m}$

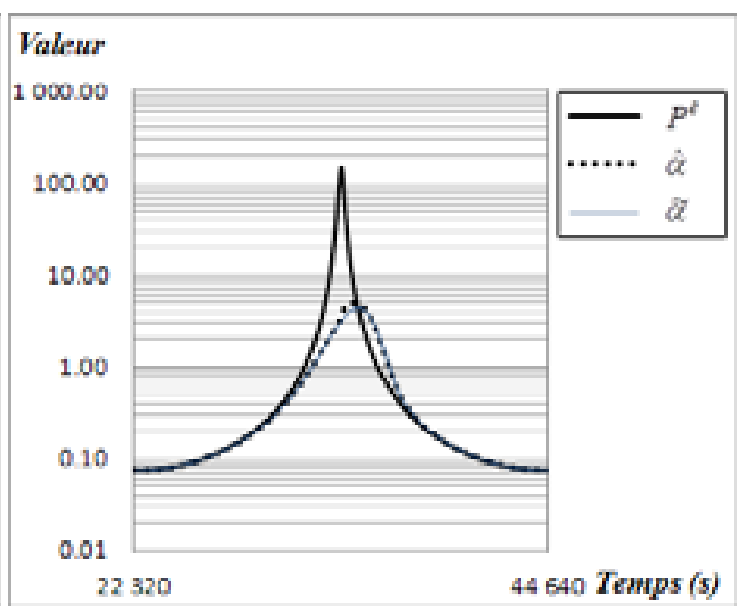

b) $d=1 \mathrm{~m}$

Figure 7. Résultats en régime non stationnaire, sans érosion et sans dépôt, pour deux cas tests définis par : $W=0,0002 \mathrm{~m} \mathrm{~s}^{-1}, U_{c-m o y}=0,02 \mathrm{~m} \mathrm{~s}^{-1}$ et $\mathrm{Ampl}=0,999$.

Le modèle phénoménologique de variation de $\tilde{\alpha}$ reste stable pour $U_{c}=0$ car dans ce cas extrême l'équation 24 devient :

$\frac{d \widetilde{\alpha}}{d t}=c_{\alpha}\left(\frac{6 W}{\kappa d}\right), s i: U_{c}=0$

Bien que pour $U_{c}=0$ le modèle soit stable, il faut signaler que pendant le laps de temps où cette condition persiste, les sédiments sont en chute libre sans diffusion turbulente, de sorte que la forme réelle des profils verticaux des matières en suspension s'écarte de celle d'une loi exponentielle, et ce d'autant plus que ce laps de temps est long.

En raison de la condition sans dépôt imposée qui est irréaliste pour $U_{c}=0$, les sédiments ont tendance, selon les modélisations 1DV, à s'accumuler dans les couches inférieures de la colonne d'eau, ce qui s'accompagne, en accord avec l'équation 26, d'un accroissement des valeurs évaluées du paramètre $\tilde{\alpha}$.

\subsection{Transport sédimentaire avec dépôt et/ou érosion}

Le dépôt et l'érosion induisent une modification dans le profil vertical de la concentration en matières en suspension qui est en général plus marquée que celle due à la non stationnarité du régime sédimentaire dépendant exclusivement des fluctuations 
Description non stationnaire de la distribution verticale des sédiments transportés en suspension par les écoulements à surface libre, en présence de dépôt et d'érosion : 9.13

des variables hydrosédimentaires. Les effets de ces deux échanges de sédiments entre le fond et l'écoulement, sont décrits par la suite.

4.2.1 Description du profil vertical de la concentration en régime non stationnaire avec dépôt

La recherche d'une description du profil vertical de la concentration en régime non stationnaire avec dépôt est basée sur les résultats de modèle numérique 1DV combinant les différentes valeurs retenues des grandeurs $U_{c-m o y}, W$ et $d$.

Les tests examinés ont été effectués avec les valeurs suivantes du paramètre $p$ de la loi de dépôt de Krone (équation 7) : 0,$125 ; 0,25 ; 0,5$ et 1 .

La première partie de cette recherche a consisté à examiner les rapports $C_{i}\left(z^{\circ}\right) / \widetilde{C}\left(z^{\circ}\right)$ à partir des résultats du modèle numérique $1 \mathrm{DV}$ en régime non stationnaire avec dépôt (figure 8). On a constaté que dans la plupart des cas ce rapport peut être décrit par une loi exponentielle $G\left(z^{\circ} ; \beta\right)=\exp \left(-\beta\left(1-z^{\circ}\right)^{2}\right)$. Pour cette raison, le modèle phénoménologique complet retenu pour décrire la concentration s'écrit comme suit :

$C\left(z^{\circ}\right)=C_{R} \exp \left(-\alpha z^{\circ}\right) \exp \left(-\beta\left(1-z^{\circ}\right)^{2}\right)$

où $C_{R}$ est une concentration de référence et $\beta$ un paramètre du modèle qui dépend des variables du problème. L'objectif est que l'écart entre $C\left(z^{\circ}\right)$ et $C_{i}\left(z^{\circ}\right)$, soit nul ou minimisé.

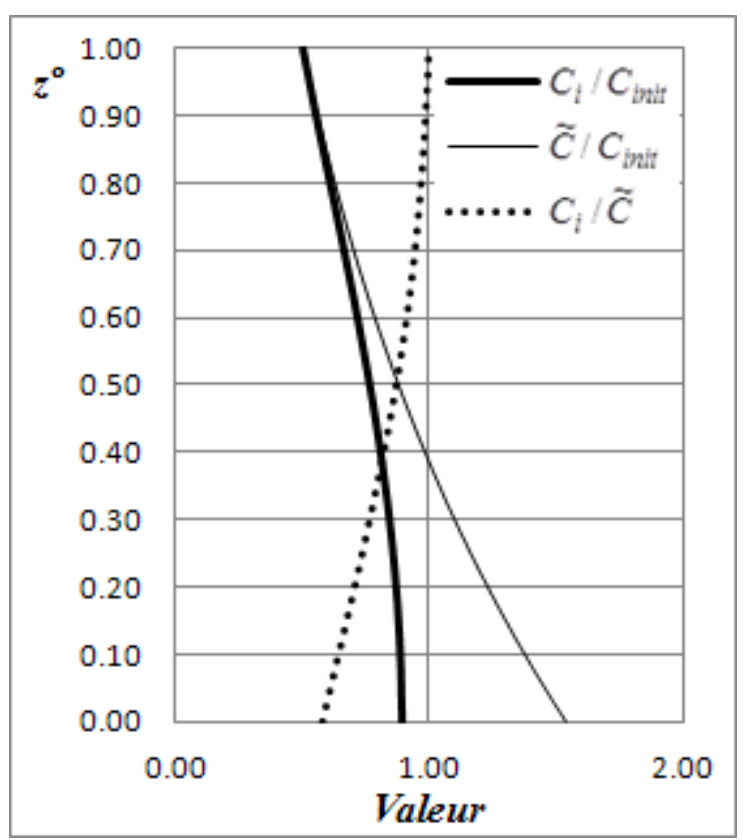

a) $P^{e}=0,98 ; p=1 ; d=8 m ; W=0,0004 \mathrm{~m} \mathrm{~s}^{-1}$

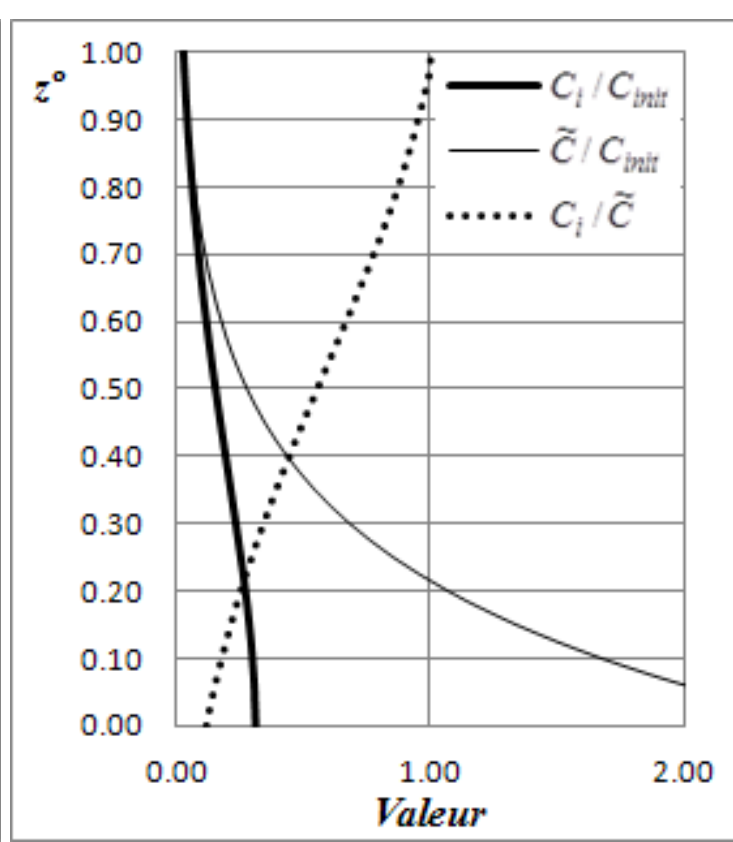

b) $P^{e}=3,9 ; p=1 ; d=8 m ; W=0,0016 \mathrm{~m} \mathrm{~s}^{-1}$

Figure 8. Exemples des profils verticaux du rapport $C_{i}\left(z^{\circ}\right) / \widetilde{C}\left(z^{\circ}\right)$ évalué à partir des résultats du modèle $1 D V$ avec dépôt (pour $C_{i}$ ) et du modèle phénoménologique ajusté en régime non stationnaire sans dépôt et sans érosion (pour $\widetilde{C}$ ). 
Sachant qu'on a retenu $\alpha=\widetilde{\alpha}$, la valeur expérimentale de la concentration de référence est calculée comme suit à partir de la valeur de la concentration en surface issue du modèle numérique $1 \mathrm{DV}$ :

$$
C_{R}=\frac{C_{i=N+1 / 2}\left(z^{\circ}=1\right)}{\exp (-\alpha)}=\frac{1,5 C_{i=N}-0,5 C_{i=N-1}}{\exp (-\alpha)}
$$

Les valeurs théoriques de $G_{i}\left(z^{\circ}\right)$ sont déterminées comme suit :

$$
G_{i}\left(z^{\circ}\right)=\frac{C_{i}\left(z^{\circ}\right)}{C_{R} \exp \left(-\alpha z^{\circ}\right)}
$$

Enfin, la valeur expérimentale de $\hat{\beta}$ a été obtenue par un ajustement de la fonction $\hat{G}\left(z^{\circ}\right)=\exp \left(-\hat{\beta}\left(1-z^{\circ}\right)^{2}\right)$ satisfaisant le critère des moindres carrés :

$\hat{\beta}=\frac{\sum_{i=1}^{N}\left(1-z^{\circ}{ }_{i}\right)^{2} \ln \left(G_{i}\right)}{\sum_{i=1}^{N}\left(1-z^{\circ}{ }_{i}\right)^{4}}$

Il a été constaté qu'une valeur stationnaire terminale $\beta_{\infty}$ peut être déterminée pour le paramètre $\hat{\beta}$ si les variables indépendantes du problème $\left(U_{c}, W\right.$ et $\left.d\right)$ restent constantes, sous condition que le paramètre $p$ de la loi de dépôt de Krone (équation 7) reste aussi constant. Dans ces conditions, le dépôt entraîne une décroissance progressive des concentrations en matières en suspension mais une stationnarité est observée pour le paramètre $\hat{\beta}$.

Les simulations avec le modèle numérique 1DV avec un dépôt imposé pendant un palier montrent que dans tous les cas la valeur de $\hat{\beta}$ tend vers une valeur terminale $\beta_{\infty}$ (voir figures 9 et 10).

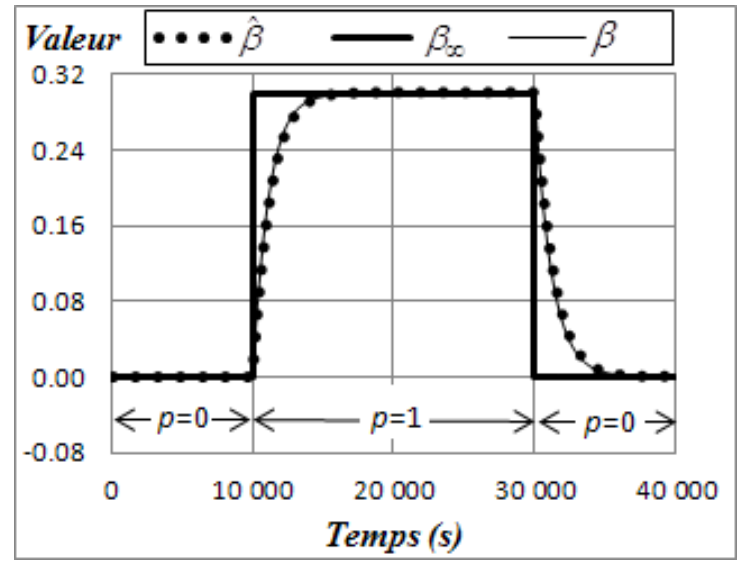

a) $\alpha=P^{e}=0,6 ; W=0,0004 \mathrm{~ms}^{-1} ; d=8 \mathrm{~m}$

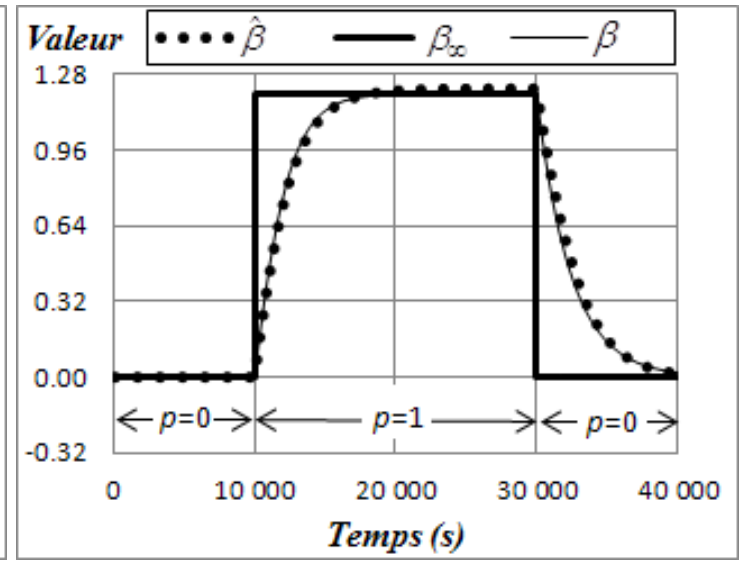

b) $\alpha=P^{e}=2,4 ; W=0,0008 \mathrm{~m} \mathrm{~s}^{-1} ; d=8 \mathrm{~m}$

Figure 9. Résultats des tests numériques $1 D \mathrm{~V}$ en régime non stationnaire avec dépôt représentés à travers les valeurs de $\hat{\beta}$. Evolution de $\beta_{\infty}=0,5 \mathrm{p} \alpha$, selon la loi ajustée pour ce paramètre et variation de $\beta$ en accord avec le modèle phénoménologique défini par l'équation 31 résolue avec la méthode predictor-corrector de Runge-Kutta. 
Description non stationnaire de la distribution verticale des sédiments transportés en suspension par les écoulements à surface libre, en présence de dépôt et d'érosion : 9.15

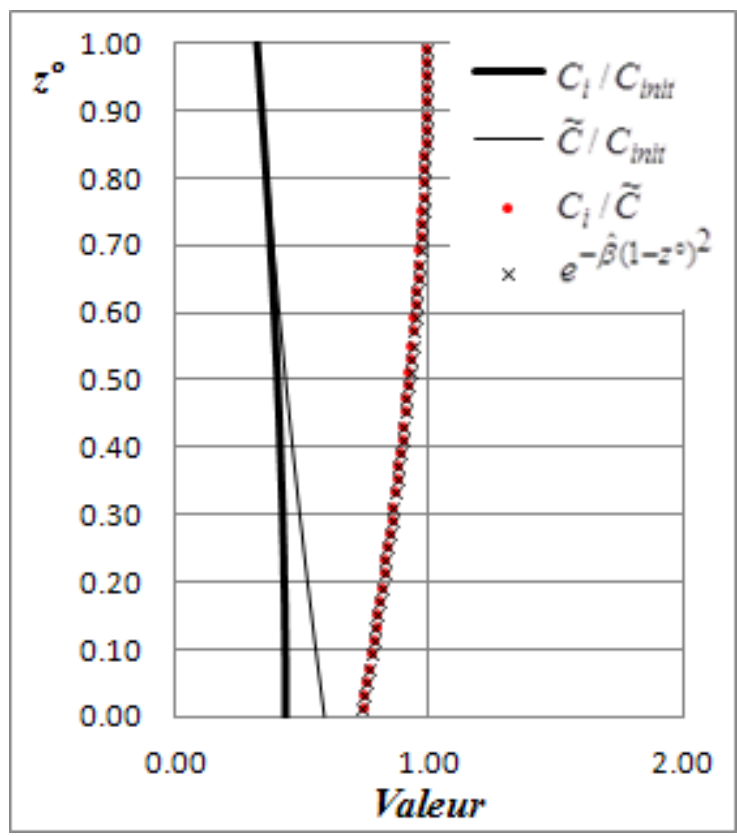

a) $\alpha=P^{e}=0,6 ; p=1 ; W=0,0004 \mathrm{~m} \mathrm{~s}^{-1} ; d=8 \mathrm{~m}$

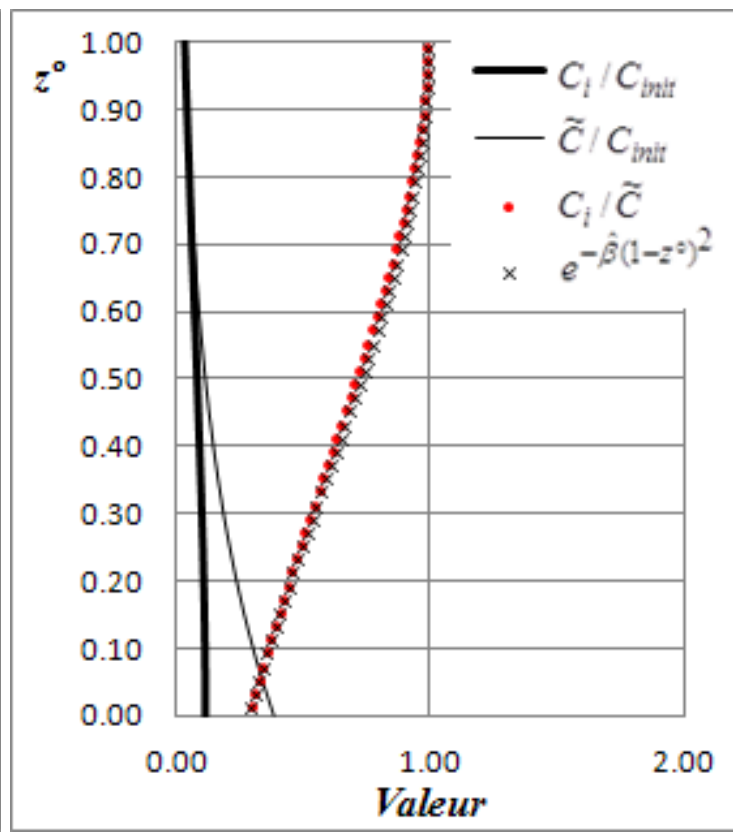

b) $\alpha=P^{e}=2,4 ; p=1 ; W=0,0008 \mathrm{~m} \mathrm{~s}^{-1} ; d=8 \mathrm{~m}$

Figure 10. Résultats des tests numériques $1 D V$ en régime non stationnaire avec dépôt représentés à travers un profil instantané de $C_{i} / C_{\text {init }}$. Profil instantané de $\widetilde{C} / C_{\text {init }}$ selon le modèle phénoménologique sans dépôt et sans érosion. Rapport $C_{i} / \widetilde{C}$ et fonction $\hat{G}\left(z^{\circ}\right)=\exp \left(-\hat{\beta}\left(1-z^{\circ}\right)^{2}\right)$ ajustée à partir de ce rapport.

Les simulations du dépôt par paliers ont permis d'évaluer en fonction des variables du problème les valeurs terminales $\beta_{\infty}$ correspondant à une stationnarité du paramètre $\hat{\beta}$. La figure 11 montre que $\beta_{\infty}$ peut être rattaché aux paramètres $p$ et $\alpha$ par : $\beta_{\infty}=0,5 p \alpha$.

De même que pour le paramètre $\widetilde{\alpha}$ lié au régime non stationnaire sans dépôt et sans érosion, l'analyse des résultats montre que le déphasage entre $\beta(t)$ et $\beta_{\infty}(t)$ dépend essentiellement du rapport $U_{c} / d$. Sur la base de cette observation le modèle retenu pour simuler la variation dans le temps de $\beta$ est la suivante :

$\frac{d \beta}{d t}=c_{\beta} \frac{U_{c}}{d}\left(\beta_{\infty}-\beta\right)$

où $c_{\beta}$ est un coefficient du modèle dont la valeur est évaluée à partir des courbes de $\hat{\beta}(t)$ issues des tests numériques 1DV avec dépôt par paliers (voir figure 9). Les valeurs retenues pour ce coefficient sont :

$c_{\beta}=\left\{\begin{array}{lll}0,667+0,3 \times \beta & , \text { si: } & D^{e f}>0 \\ 0,667 & , \text { si: } & D^{e f}=0\end{array}\right.$

Il est intéressant de remarquer qu'en régime stationnaire terminal pour les paramètres $\alpha$ et $\beta$, le modèle phénoménologique développé dans le cadre de cette étude s'écrit comme suit :

$\left.C\left(z^{\circ}\right)\right|_{\alpha_{\infty}, \beta_{\infty}(p, \alpha)} \approx C_{R} \exp \left(-P^{e} z^{\circ}\right) \exp \left(-0,5 p P^{e}\left(1-z^{\circ}\right)^{2}\right)$ 
Ainsi, cette approche du profil vertical de la concentration est compatible avec les conditions de frontière du problème au fond $(z=0)$ et en surface $(z=d)$. En effet :

$$
\begin{aligned}
& \left(-\left.W C\right|_{\alpha_{\infty}, \beta_{\infty}(p, \alpha)}-K_{z} \frac{\left.\partial C\right|_{\alpha_{\infty}, \beta_{\infty}(p, \alpha)}}{\partial z}\right)_{z=0}=\left(-\left.p W C\right|_{\alpha_{\infty}, \beta_{\infty}(p, \alpha)}\right)_{z=0} \\
& \left(-\left.W C\right|_{\alpha_{\infty}, \beta_{\infty}(p, \alpha)}-K_{z} \frac{\left.\partial C\right|_{\alpha_{\infty}, \beta_{\infty}(p, \alpha)}}{\partial z}\right)_{z=d}=0
\end{aligned}
$$

Cependant, on doit signaler que lorsque les paramètres $\alpha$ et $\beta$ ne sont pas stationnaires, ces conditions de frontière ne sont pas satisfaites rigoureusement, si bien que dans ces cas le modèle inclut une incertitude qui peut être étudiée à travers les erreurs inhérentes aux résultats du modèle phénoménologique. L'étude de ces erreurs se fera dans la section 4.2 .3 où le dépôt et l'érosion sont simulés au cours d'un même test.

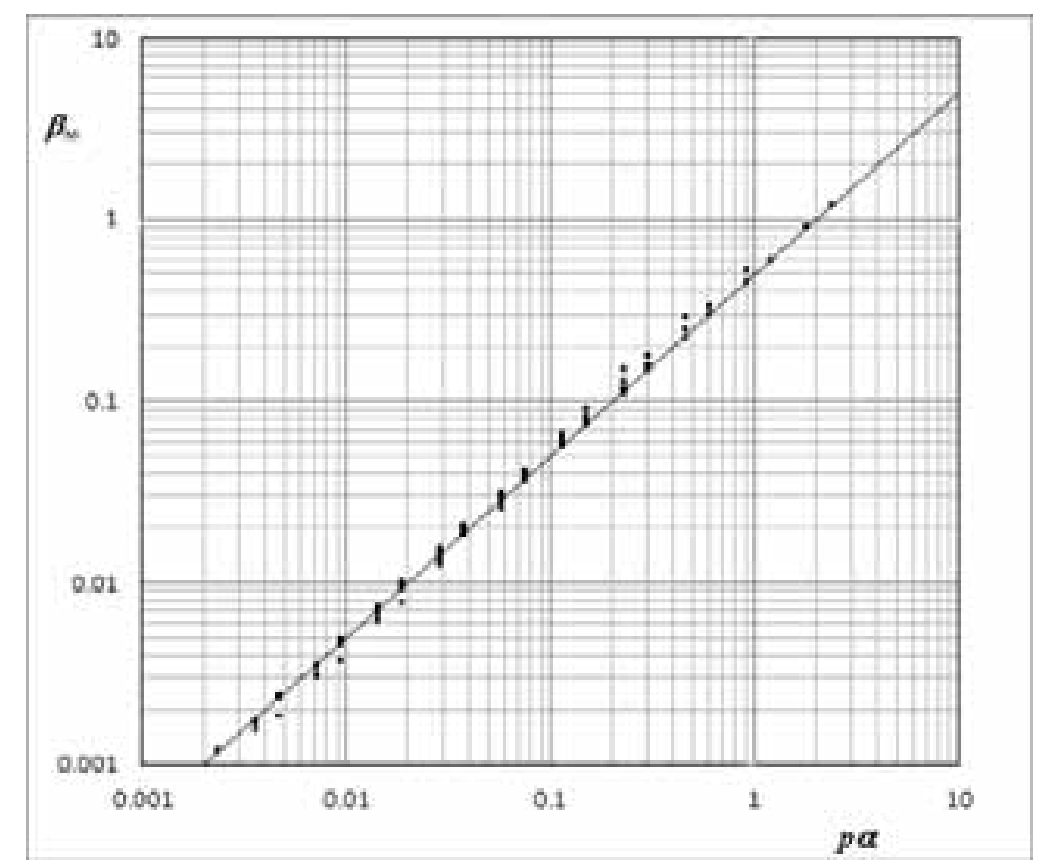

Figure 11. Relation entre le paramètre $\beta_{\infty}$ et le produit $p \times \alpha$ (516 points expérimentaux).

Le trait continu correspond à la loi ajustée $\beta_{\infty}=0,5 \mathrm{p} \alpha$.

4.2.2 Description du profil vertical de la concentration en régime non stationnaire avec érosion

Le paramètre $\beta$ tenant compte de l'effet du dépôt sur le profil vertical de la concentration sert également à tenir compte de l'érosion. On constate que dans ce cas aussi une valeur stationnaire terminale $\beta_{\infty}$ peut être déterminée pour le paramètre $\hat{\beta}$ si les variables indépendantes du problème $\left(U_{c}, W\right.$ et $\left.d\right)$ restent constantes, mais seulement à condition qu'un paramètre défini par $q=E^{e f} /\left(C_{0} W_{0}\right)$ reste aussi constant. 
Description non stationnaire de la distribution verticale des sédiments transportés en suspension par les écoulements à surface libre, en présence de dépôt et d'érosion : 9.17

On doit signaler que cette dernière condition n'est pas "naturelle" dans les processus sédimentaires car en général $E^{e f}$ est indépendant de $C_{0} W_{0}$. En effet, il est admis que le taux d'érosion effectif $E^{e f}$ dépend principalement des contraintes hydrodynamiques érosives et de la tenue des sédiments déposés à ces contraintes.

Cependant, les modélisations de l'érosion par paliers avec le paramètre $q$ constant fournissent la méthode numérique la plus précise disponible pour évaluer $\beta_{\infty}$ et $c_{\beta}$ (voir figures 12 et 13). Les tests ont permis d'évaluer $c_{\beta}=0,667$.

En régime stationnaire terminal pour les paramètres $\alpha$ et $\beta$, une relation $\beta_{\infty}=-0,5 \times q \alpha$ est compatible avec les conditions de frontière au fond et en surface. Les résultats obtenus du modèle numérique 1DV avec érosion par paliers ont montré que cette relation n'est valable que pour des valeurs de $q \alpha$ inférieures à 0,1 (voir figure 14), bien qu'au-dessus de cette valeur, $\beta_{\infty}$ reste fortement corrélé à $q \alpha$. L'approche retenue est la suivante :

$\beta_{\infty}=\left\{\begin{array}{lll}-0,50 \times(q \alpha) & , \text { si }: & q \alpha<0,10 \\ -0,72 \times(q \alpha)^{1,15} & , \text { si }: & q \alpha>0,10\end{array}\right.$

Nous devons signaler qu'au cours de cette étude nous avons constaté que dans certains cas, avec érosion notamment, les résultats du modèle numérique $1 \mathrm{DV}$ avec $N=20 \mathrm{sont}$ moins précis que ceux issus du modèle phénoménologique développé dans cet article. Dans cet étude ce problème a été résolu en prenant $N=50$, ou $N=100$ dans certains cas.

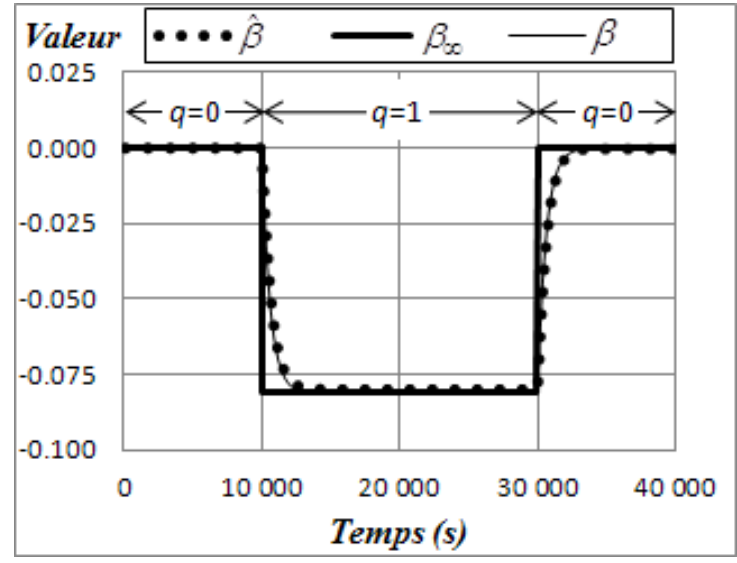

a) $\alpha=P^{e}=0,15 ; W=0,0004 \mathrm{~ms}^{-1} ; d=16 \mathrm{~m}$

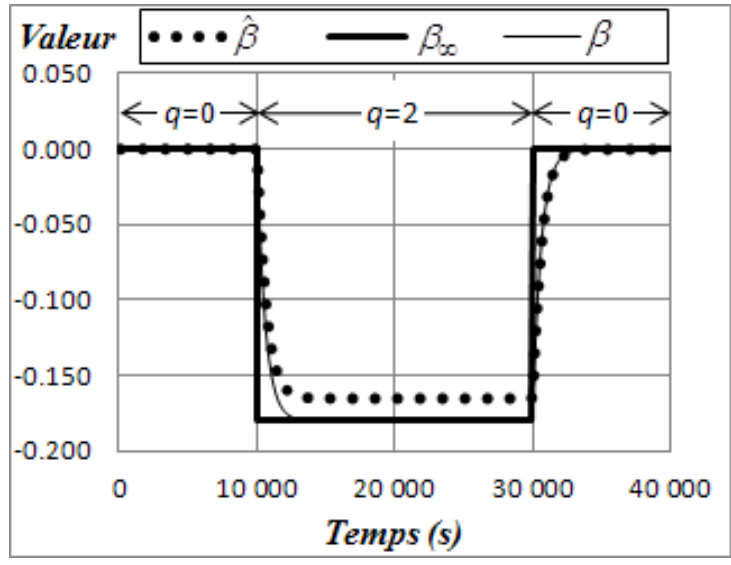

b) $\alpha=P^{e}=0,15 ; W=0,0004 \mathrm{~ms}^{-1} ; d=16 \mathrm{~m}$

Figure 12. Résultats des tests numériques $1 D V$ en régime non stationnaire avec érosion représentés à travers les valeurs de $\hat{\beta}$. Evolution de $\beta_{\infty}(q, \alpha)$ selon la loi ajustée pour ce paramètre et variation de $\beta$ en accord avec le modèle phénoménologique défini par

l'équation 31 résolue avec la méthode predictor-corrector de Runge-Kutta.

\subsubsection{Simulations en régime non stationnaire avec érosion et dépôt}

Dans cette section sont présentés des résultats obtenus à partir des modélisations 1DV correspondant à des simulations de la dynamique verticale des matières en suspension en régime non stationnaire avec érosion et dépôt. 


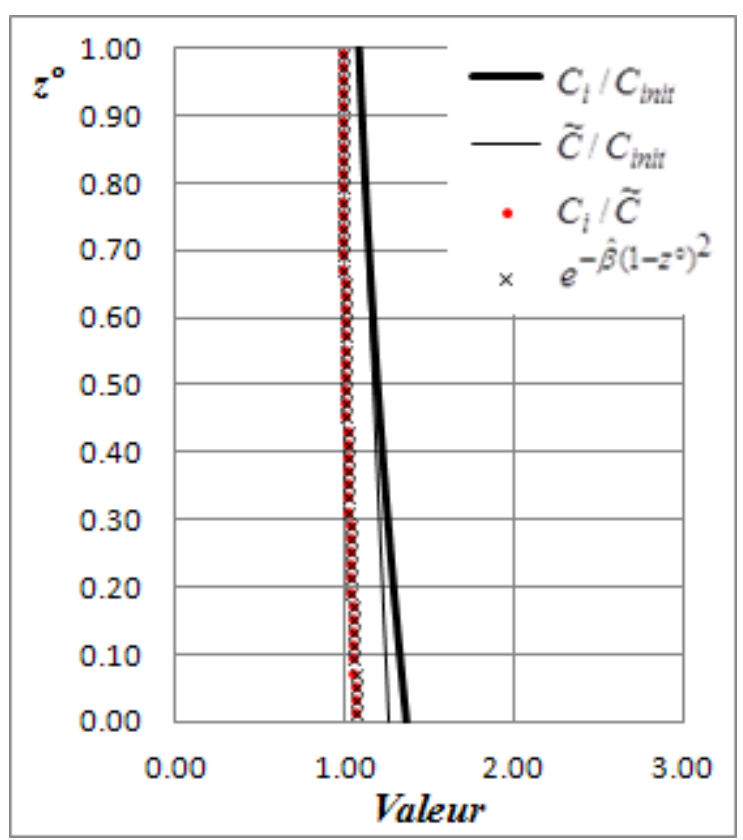

a) $\alpha=P^{e}=0,15 ; q=1 ; W=0,0004 \mathrm{~m} \mathrm{~s}^{-1} ; d=16 \mathrm{~m}$

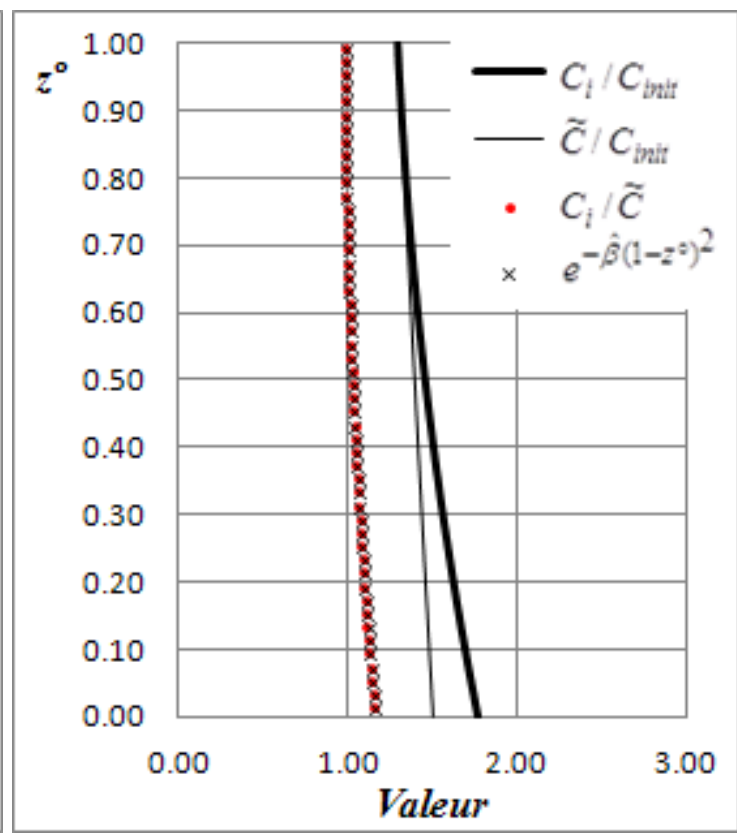

b) $\alpha=P^{e}=0,15 ; q=2 ; W=0,0004 \mathrm{~m} \mathrm{~s}^{-1} ; d=16 \mathrm{~m}$

Figure 13. Résultats des tests numériques $1 D V$ en régime non stationnaire avec érosion représentés à travers un profil instantané de $C_{i} / C_{\text {init. }}$. Profil instantané de $\widetilde{C} / C_{\text {init }}$ selon le modèle phénoménologique sans dépôt et sans érosion. Rapport $C_{i} / \widetilde{C}$ et fonction $\hat{G}\left(z^{\circ}\right)=\exp \left(-\hat{\beta}\left(1-z^{\circ}\right)^{2}\right)$ ajustée à partir de ce rapport.

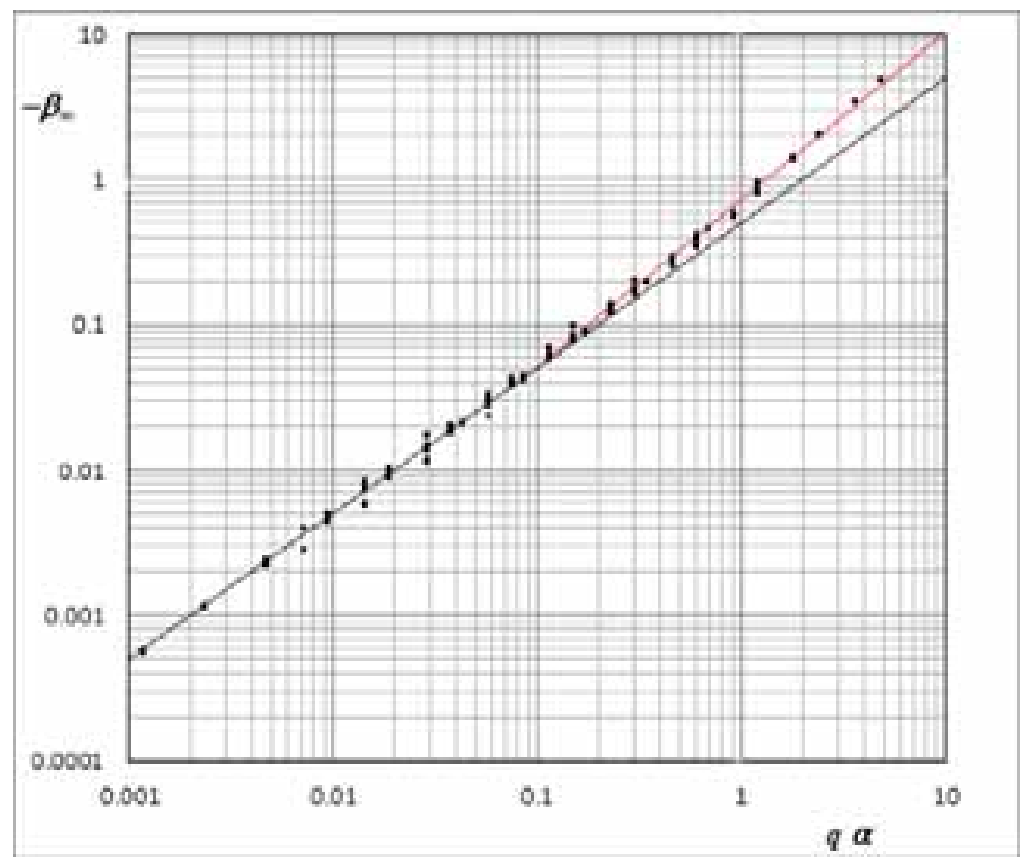

Figure 14. Relation entre $-\beta_{\infty}$ et le produit $q \times \alpha$ (596 points expérimentaux).

Le trait en noir correspond à $\beta_{\infty}=-0,5(q \alpha)$, et le trait en rouge à la loi ajustée pour les valeurs de qa supérieures à 0,10 (voir équation 35 ). 
Description non stationnaire de la distribution verticale des sédiments transportés en suspension par les écoulements à surface libre, en présence de dépôt et d'érosion : 9.19

Ces résultats sont comparés avec ceux obtenus avec le modèle phénoménologique développé dans cet article. Les résultats de deux simulations numériques test sont décrits par la suite. Ces simulations sont désignées Cas 1 et Cas 2.

Les valeurs des variables du problème relatives au Cas 1 étudié sont rassemblées dans le tableau 1. En raison des valeurs imposées, le nombre sans dimension $\left(U_{c-m o y} \times T / d\right)$ est relativement élevé, si bien qu'en l'absence des phénomènes de dépôt et d'érosion, le régime sédimentaire serait quasi stationnaire (voir équation 25 ).

Les résultats du modèle 1DV qui sont présentés sur la figure 15, montrent, en cohérence avec les résultats du modèle phénoménologique, que les paramètres de ce dernier sont quasi stationnaires. On constate qu'à tout instant les paramètres $\alpha$ et $\beta$ sont proches de leurs valeurs terminales instantanées respectives $\alpha_{\infty}$ et $\beta_{\infty}$.

Pendant les intervalles de temps où le profil vertical de la concentration n'est pas affecté par les échanges avec le fond (érosion et dépôt), on constate que le signal $\hat{\alpha}$ obtenu à partir des résultats des modélisations numériques $1 \mathrm{DV}$, est bien approché par le signal du modèle phénoménologique $\alpha=\widetilde{\alpha}$. En présence de dépôt et d'érosion les signaux $\alpha$ et $\hat{\alpha}$ s'écartent, ce qui est dû à une modification de la forme du profil vertical de la concentration par rapport à sa forme sans dépôt et sans érosion.

Pendant les intervalles de temps où la dynamique sédimentaire est affectée par l'érosion ou par le dépôt, la modification de la forme du profil vertical de la concentration est contrôlée par le paramètre $\beta$. Un très bon accord entre $\beta$ et $\hat{\beta}$ implique que la précision des résultats du modèle phénoménologique est excellente dans ce cas étudié.

Pour mieux caractériser la précision globale du modèle phénoménologique, on a calculé les erreurs relatives au fond $e\left(z^{\circ}=0\right)$ comme suit :

$e\left(z^{\circ}=0\right)=\frac{C\left(z^{\circ}=0\right)}{C_{i}\left(z^{\circ}=0\right)}-1=\frac{C_{R} \exp (-\beta)}{C_{i=1-1 / 2}\left(z^{\circ}=0\right)}-1=\frac{C_{R} \exp (-\beta)}{1,5 C_{i=1}-0,5 C_{i=2}}-1$

Sachant que la concentration moyenne sur la verticale est donnée par :

$\bar{C}=\int_{0}^{1} C_{i} d z^{\circ} \approx \int_{0}^{1} C d z^{\circ}=\int_{0}^{1} C_{R} \exp \left(-\alpha z^{\circ}\right) \exp \left(-\beta\left(1-z^{\circ}\right)^{2}\right) d z^{\circ}$

La valeur de $C_{R}$ a été calculée par:

$$
C_{R}=\frac{\sum_{i=1}^{N} C_{i} \Delta z^{\circ}}{\sum_{i=1}^{N} \exp \left(-\alpha z^{\circ}{ }_{i}\right) \exp \left(-\beta\left(1-z^{\circ}{ }_{i}\right)^{2}\right) \Delta z^{\circ}}
$$

On observe que le signal de $e(0)$ comporte des valeurs de pic modérés liés à un début et une fin intempestifs des phénomènes d'érosion et de dépôt. Par ailleurs, les calculs montrent que les valeurs maximales de $\left|e\left(z^{\circ}=0\right)\right|$ sont inférieures à 0,006 ce qui confirme la très bonne qualité des résultats du modèle phénoménologique dans ce cas. 
Tableau 1. Valeurs des variables du problème définissant le Cas 1 étudié.

\begin{tabular}{ll}
\hline Variable & Symbole et valeur \\
\hline Profondeur & $d=2,00 \mathrm{~m}$ \\
Vitesse de chute & $W=0,0002 \mathrm{~m} \mathrm{~s}^{-1}$ \\
Valeur moyenne de la vitesse de cisaillement & $U_{c-\text { moy }}=0,04 \mathrm{~m} \mathrm{~s}^{-1}$ \\
Période du signal $U_{c}$ & $T=22320 \mathrm{~s}$ \\
Amplitude relative du signal $U_{c}$ & $A m p l=0,80$ \\
Condition critique de dépôt & $U_{c}($ critique de dépôt $)=0,50 \times U_{c-m o y}$ \\
Paramètre de la loi de Krone de dépôt & $p=0,50$ \\
Condition critique d'érosion & $U_{c}($ critique d'érosion $)=1,70 \times U_{c-m o y}$ \\
Taux d'érosion effectif adimensionnel & $E^{e f} /\left(W \times \overline{C_{\text {init }}}\right)=1,00$ \\
\hline
\end{tabular}

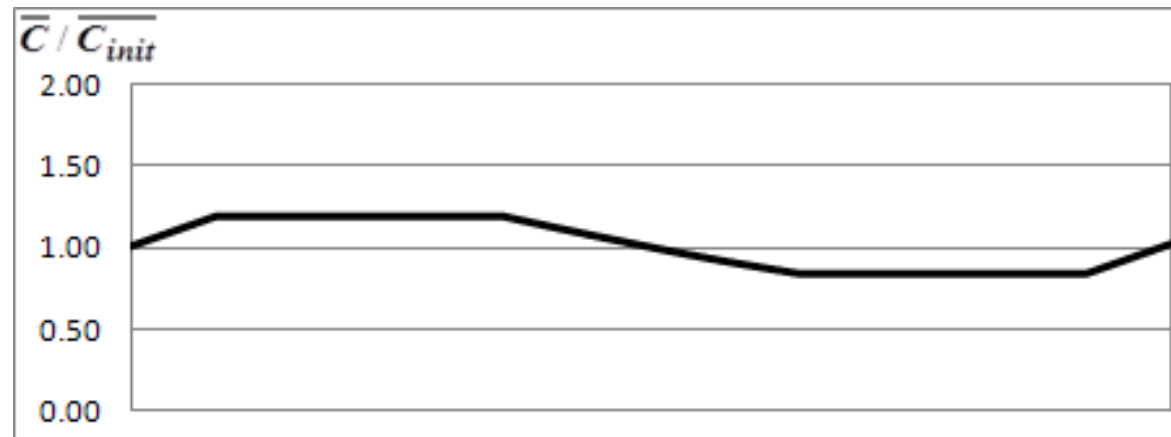

22320

44640 Temps (s)

\section{Valeur}

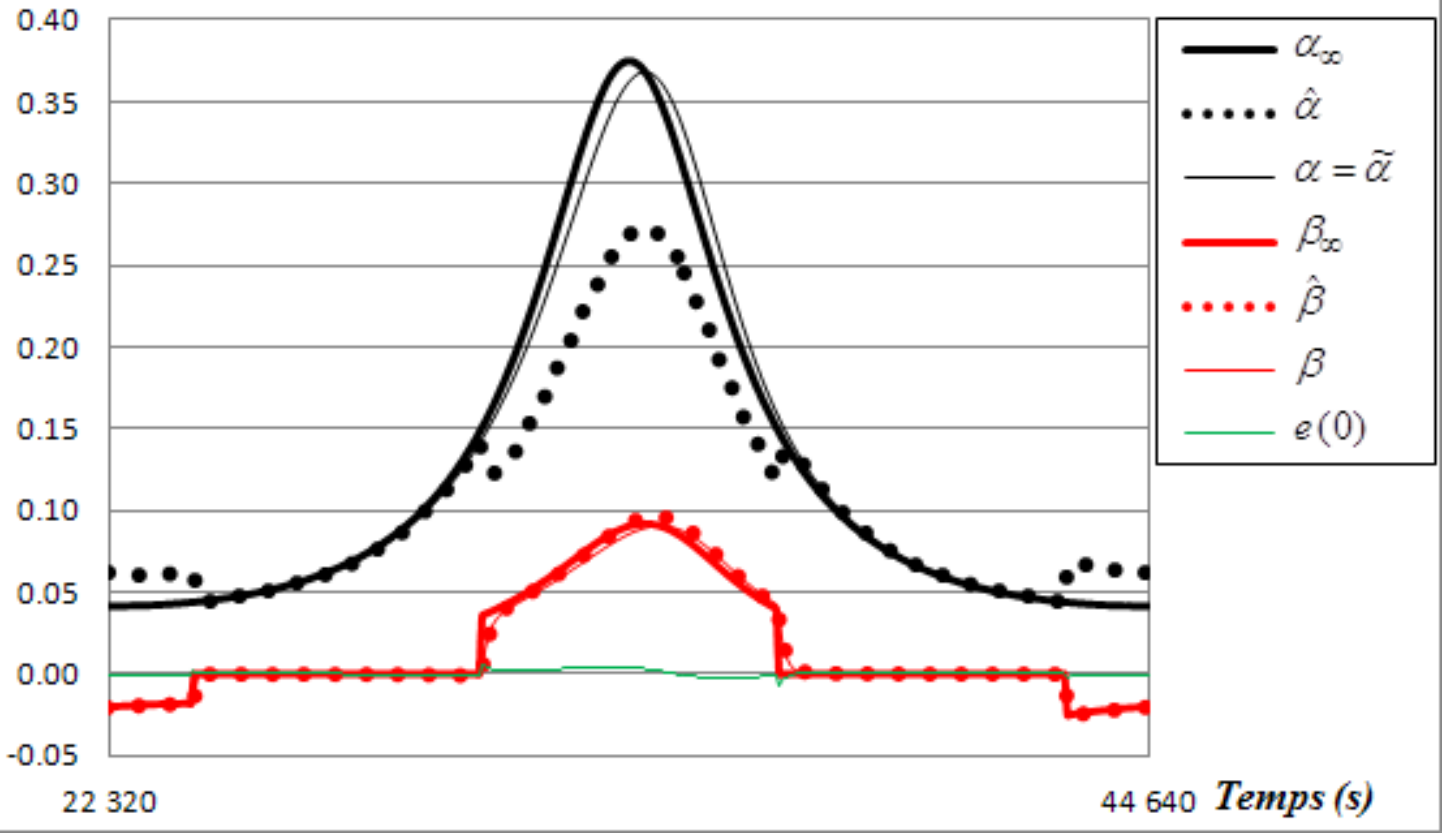

Figure 15. Résultats des modélisations correspondant au Cas 1 étudié défini par les valeurs des variables rassemblées dans le tableau 1. Cette figure présente la variation dans le temps de la concentration moyenne sur la verticale et des paramètres du modèle phénoménologique décrivant le profil vertical des matières en suspension. 
Description non stationnaire de la distribution verticale des sédiments transportés en suspension par les écoulements à surface libre, en présence de dépôt et d'érosion : 9.21

Les valeurs des variables du problème relatives au Cas 2 étudié sont rassemblées dans le tableau 2 et les résultats correspondants sur la figure 16. On constate que les valeurs de $\alpha$ et $\alpha_{\infty}$ sont en général différentes, mais ces deux valeurs sont presque identiques pour les faibles valeurs de $\alpha_{\infty}=P^{e}$. Les valeurs de $\beta$ sont proches de celles de $\beta_{\infty}$ pendant les phases d'érosion qui coïncident avec une valeur faible de $P^{e}$ alors que pendant les phases de dépôt où $P^{e}$ atteint ses valeurs maximales, les valeurs de $\beta$ et $\beta_{\infty}$ sont en général différentes.

En bref, dans ce Cas 2 étudié, les paramètres adimensionnels du modèle phénoménologique se rapprochent de leurs valeurs terminales théoriques pour les valeurs faibles de $P^{e}$ qui correspondent aux fortes valeurs de $U_{c}$, grandeur qui contrôle les transferts de masse verticaux liés à la turbulence.

Les valeurs extrêmes de $e(0)$ sont plus importantes que dans le Cas 1 . En effet, en valeur absolue l'erreur relative atteint une valeur maximale de 0,10 , ce qui nous semble bien acceptable.

La figure 17 représente des profils verticaux de la concentration adimensionnés par la concentration initiale des modélisations. Quatre séquences de profils sont montrées correspondant à quatre fenêtres d'observation. Les instants $t_{o}$ de ces fenêtres sont définis par :

- Début de la période de dépôt.

- Fin de la période de dépôt.

- Début de la période d'érosion.

- Fin de la période d'érosion.

D'une façon générale, il y a un très bon accord entre les résultats numériques du modèle théorique 1DV et ceux du modèle phénoménologique. Dans tous les cas étudiés, une bonne convergence de ce dernier est vérifiée, bien que pendant la période qui suit le début du dépôt, un léger retard pour la convergence à proximité du fond soit constaté.

Tableau 2. Valeurs des variables du problème définissant le Cas 2 étudié.

\begin{tabular}{ll}
\hline Variable & Symbole et valeur \\
\hline Profondeur & $d=8,00 \mathrm{~m}$ \\
Vitesse de chute & $W=0,0012 \mathrm{~m} \mathrm{~s}^{-1}$ \\
Valeur moyenne de la vitesse de cisaillement & $U_{c-\text { moy }}=0,04 \mathrm{~m} \mathrm{~s}^{-1}$ \\
Période du signal $U_{c}$ & $T=22320 \mathrm{~s}$ \\
Amplitude relative du signal $U_{c}$ & Ampl=0,80 \\
Condition critique de dépôt & $U_{c}($ critique de dépôt $)=0,50 \times U_{c-m o y}$ \\
Paramètre de la loi de Krone de dépôt & $p=1,00$ \\
Condition critique d'érosion & $U_{c}($ critique d'érosion $)=1,70 \times U_{c-m o y}$ \\
Taux d'érosion effectif adimensionnel & $E^{e f} /\left(W \times \overline{C_{\text {init }}}\right)=2,50$ \\
\hline
\end{tabular}




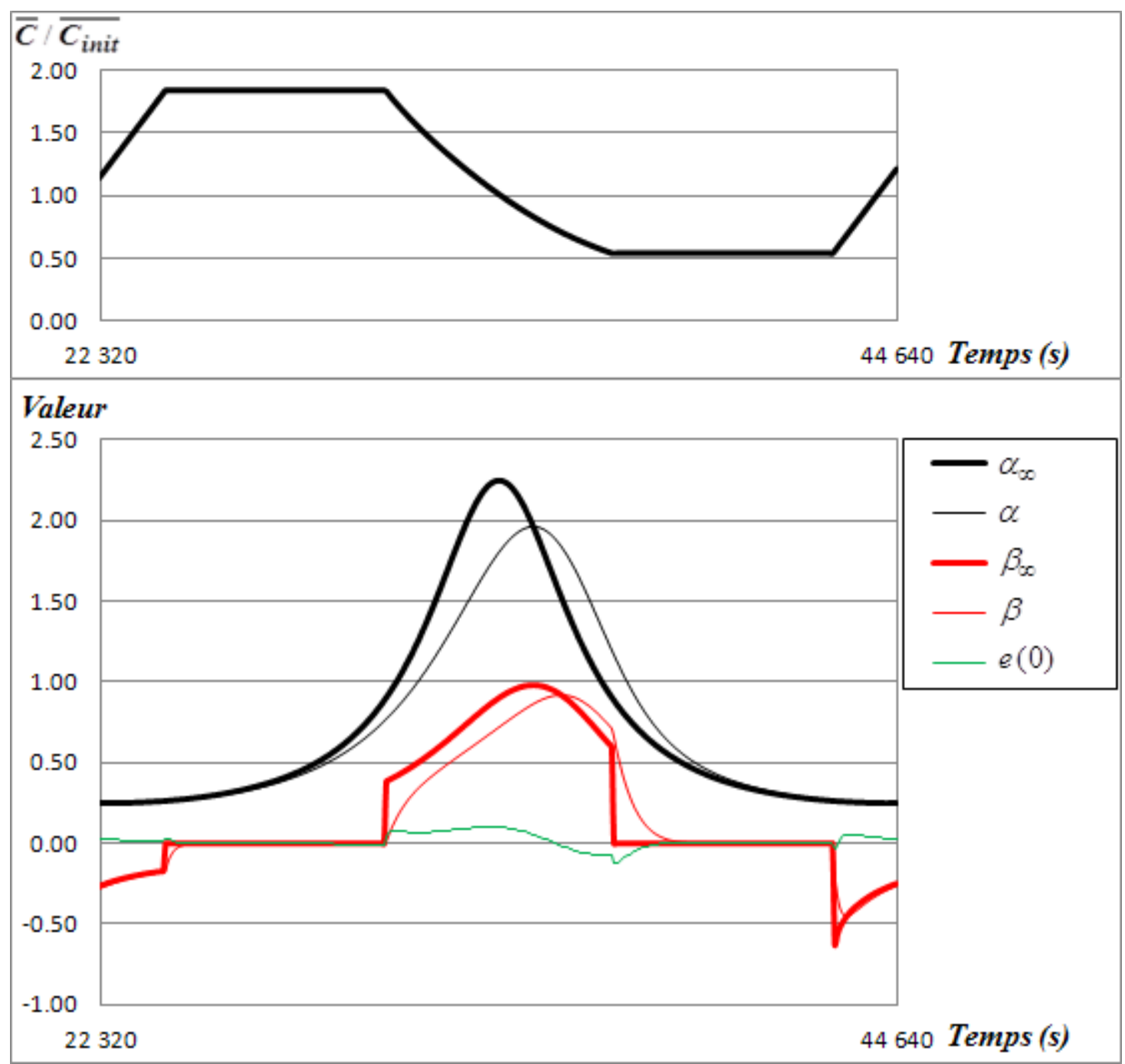

Figure 16. Résultats des modélisations correspondant au Cas 2 étudié défini par les valeurs des variables rassemblées dans le tableau 2. Cette figure présente la variation dans le temps de la concentration moyenne sur la verticale et des paramètres adimensionnels du modèle phénoménologique décrivant le profil vertical des matières en suspension.

\section{Synthèse du modèle phénoménologique de convection-diffusion des matières en suspension}

Dans les écoulements à surface libre étudiés dans cet article, les matières en suspension sont transportées horizontalement par le courant. Dans ces conditions, les modèles phénoménologiques développés pour les paramètres alpha et bêta peuvent s'appliquer seulement si l'on suit les sédiments dans leur parcours horizontal. En coordonnées d'Euler cela implique la nécessité d'inclure des termes de convection horizontale dans les équations du modèle. 
Description non stationnaire de la distribution verticale des sédiments transportés en suspension par les écoulements à surface libre, en présence de dépôt et d'érosion : 9.23

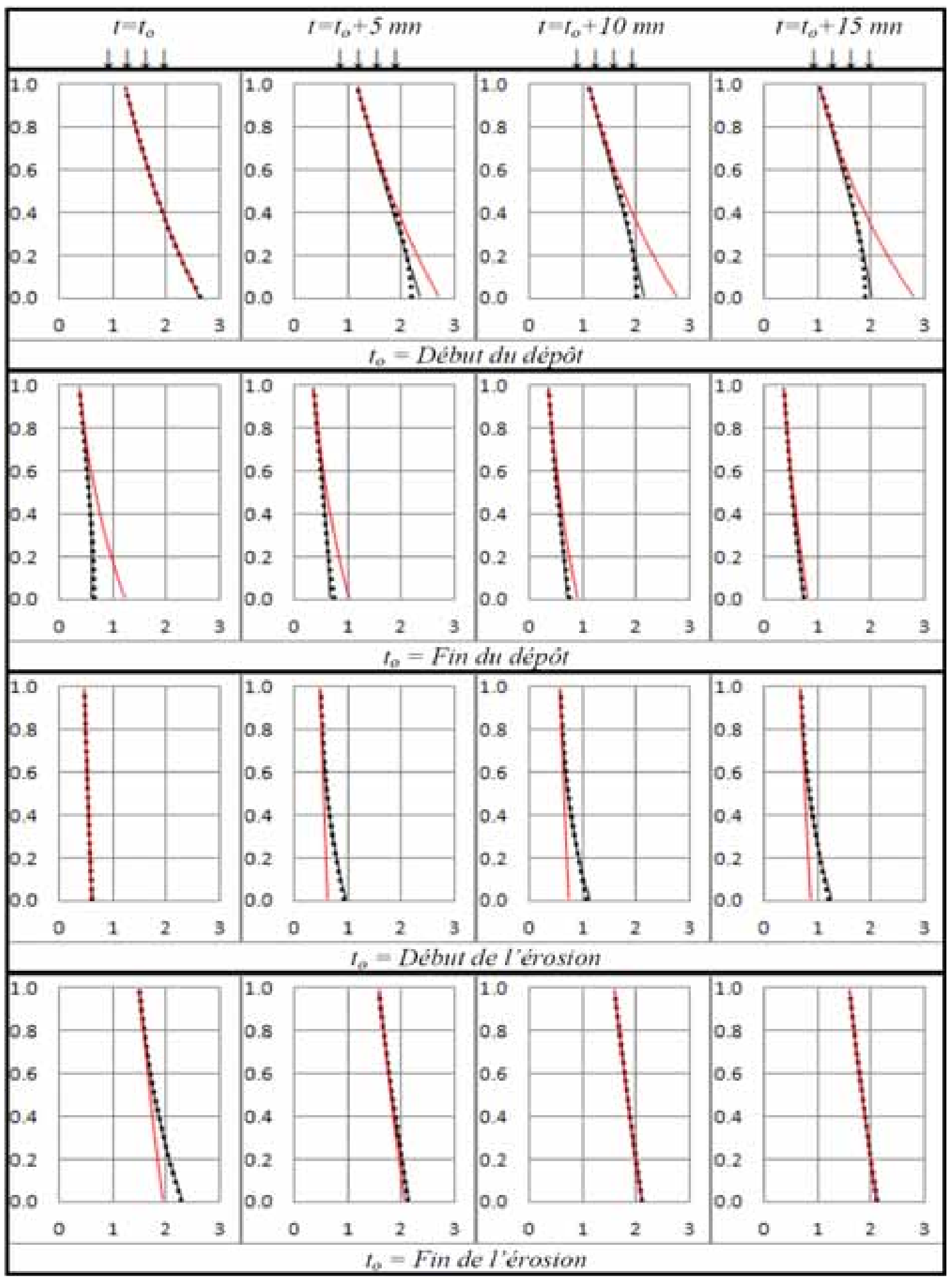

Figure 17. Concentrations adimensionnées par la concentration initiale en abscisses, en fonction de $z^{\circ}$ en ordonnées. En pointillés noirs : résultats numériques du modèle $1 D V$. En trait noir : résultats du modèle phénoménologique. En trait rouge : loi exponentielle avec alpha comme seul paramètre adimensionnel. 
Les équations finales retenues pour le modèle $2 \mathrm{DH}$ de transport de sédiments en suspension par un écoulement sont présentées par la suite. Tout d'abord l'équation de transport régissant la concentration moyenne sur la verticale $\bar{C}$ :

$\frac{\partial \bar{C}}{\partial t}+\bar{V}_{x} \frac{\partial \bar{C}}{\partial x}+\bar{V}_{y} \frac{\partial \bar{C}}{\partial y}=\frac{\partial}{\partial x}\left(\bar{K}_{x} \frac{\partial \bar{C}}{\partial x}\right)+\frac{\partial}{\partial y}\left(\bar{K}_{y} \frac{\partial \bar{C}}{\partial y}\right)+\frac{E^{e f}-D^{e f}}{d}$

où $\bar{V}_{x}$ et $\bar{V}_{y}$ sont les composantes de la vitesse moyenne sur la verticale selon les directions $O x$ et $O y$ respectivement.

Le profil vertical de la concentration est décrit par l'équation 27, reprise ci-dessous :

$C\left(z^{\circ}\right)=C_{R} \exp \left(-\alpha z^{\circ}\right) \exp \left(-\beta\left(1-z^{\circ}\right)^{2}\right)$

D'après l'équation 38 , la concentration de référence du profil $C_{R}$ est reliée à $\bar{C}$ par :

$\bar{C}=C_{R} \int_{0}^{1} \exp \left(-\alpha z^{\circ}\right) \exp \left(-\beta\left(1-z^{\circ}\right)^{2}\right) d z^{\circ}$

La concentration au fond $C_{0}$, dont la valeur sert à paramétrer le taux de dépôt effectif $D^{e f}$, est reliée à $C_{R}$ par :

$C_{0}=C_{R} \exp (-\beta)$

Le paramètre $\alpha=\tilde{\alpha}$, d'après l'équation 24 avec prise en compte de la convection horizontale, est régi par :

$\frac{\partial \alpha}{\partial t}+\bar{V}_{x} \frac{\partial \alpha}{\partial x}+\bar{V}_{y} \frac{\partial \alpha}{\partial y}=c_{\alpha} \frac{U_{c}}{d}\left(\alpha_{\infty}-\alpha\right)$

où $\alpha_{\infty}=P^{e}$ est la valeur terminale du paramètre $\alpha$ en régime stationnaire et $c_{\alpha} \approx 0,667$ un coefficient du modèle. Selon l'équation 31 avec prise en compte de la convection horizontale, le paramètre $\beta$ est régi par :

$\frac{\partial \beta}{\partial t}+\bar{V}_{x} \frac{\partial \beta}{\partial x}+\bar{V}_{y} \frac{\partial \beta}{\partial y}=c_{\beta} \frac{U_{c}}{d}\left(\beta_{\infty}-\beta\right)$

où $\beta_{\infty}$ est la valeur terminale du paramètre $\beta$, laquelle dépend des échanges solides entre le fond et la colonne d'eau, échanges paramétrés par $D^{e f}$ et $E^{e f}$.

En cas de dépôt effectif $\left(D^{e f}>0 ; E^{e f}=0\right)$ :

$$
\beta_{\infty}=0,50 p \alpha \quad \text {,et: } \quad c_{\beta}=0,667+0,3 \times \beta
$$

En cas d'érosion effective $\left(D^{e f}=0 ; E^{e f}>0\right)$ :

$\beta_{\infty}=\left\{\begin{array}{lll}-0,50 \times(q \alpha) & , \text { si }: & q \alpha<0,10 \\ -0,72 \times(q \alpha)^{1,15} & , \text { si }: & q \alpha>0,10\end{array} \quad\right.$,et $: \quad c_{\beta}=0,667$

En cas d'échanges effectifs nuls entre le fond et l'écoulement $\left(D^{e f}=0 ; E^{e f}=0\right)$ :

$\beta_{\infty}=0 \quad$,et: $\quad c_{\beta}=0,667$

5.1 Modèle de convection-diffusion verticale à deux équations

Ce modèle doit être utilisé quand $\alpha$ et $\beta$ sont en général différents de $\alpha_{\infty}$ et de $\beta_{\infty}$, respectivement. 
Description non stationnaire de la distribution verticale des sédiments transportés en suspension par les écoulements à surface libre, en présence de dépôt et d'érosion : 9.25

Si les actions hydrodynamiques paramétrées par $\left|U_{c}\right|$ ont une périodicité $T$, cela correspond à un nombre sans dimensions $\left(U_{c-m o y} \times T / d\right)<<500$. Les paramètres adimensionnels du modèle doivent être simulés par les équations $40 \mathrm{~A}$ et $40 \mathrm{~B}$. Les résultats obtenus sont alors fiables à tout moment.

\subsection{Modèle de convection-diffusion verticale à une équation}

Dans certains problèmes à tout instant on a $\beta \approx \beta_{\infty}$, alors qu'en général $\alpha \neq \alpha_{\infty}$. Dans ce cas on retient $\beta=\beta_{\infty}$, mais $\alpha$, qui est en décalage sur $\alpha_{\infty}$, doit être modélisé avec l'équation $40 \mathrm{~A}$. Le fait de retenir $\beta=\beta_{\infty}$ peut induire quelques erreurs significatives pendant des laps de temps courts suivant la fin des périodes de dépôt et d'érosion (voir figures 15 et 16). Ce cas correspond à $\left(U_{c-m o y} \times T / d\right) \approx 500$

\subsection{Modèle de convection-diffusion verticale à zéro équation}

Dans ce cas on admet la stationnarité des paramètres $\alpha$ et $\beta$. On retient alors $\alpha=\alpha_{\infty}$ et $\beta=\beta_{\infty}$. Ce cas correspond à $\left(U_{c-m o y} \times T / d\right)>\sim 500$ avec des périodes très courtes ou inexistantes avec faibles valeurs de $U_{c}$. Les résultats obtenus pour $C_{0} / \bar{C}$ sont alors très proches de ceux calculés avec la formulation de TEETER (1986), (équation 14) et pratiquement identiques pour des valeurs de $p$ proches de 1 .

\section{Conclusions}

L'étude des formes des profils verticaux de la concentration permet de tirer deux conclusions générales indépendantes du modèle phénoménologique développé dans ce manuscrit :

- En régime sédimentaire stationnaire, les solutions analytiques ou par intégration numérique des équations régissant le problème, permettent de décrire les profils de $C / C_{0}$ par une fonction $F\left(z^{\circ} ; \alpha_{\infty}\right)$ qui définit l'équation générale de ces profils, où $\alpha_{\infty}$ est un paramètre adimensionnel qui dépend du rapport $W / U_{c}$.

En régime non stationnaire, lorsque des conditions sans dépôt et sans érosion sont imposées dans les modélisations, à tout moment les profils de $C / C_{0}$ convergent vers $F\left(z^{\circ} ; \alpha_{\infty}\right)$. Bien que la convergence réelle soit plus rapide au fond et en surface que sur le reste de la colonne d'eau, dans la plupart des cas les profils de $C / C_{0}$ sont assez bien modélisés par la même fonction $F\left(z^{\circ} ; \alpha\right)$, avec la valeur du paramètre $\alpha$ qui tend toujours vers la valeur instantanée de $\alpha_{\infty}$. Selon ces mêmes modélisations, le seul écart significatif de cette équation générale du profil de $C / C_{0}$ est observé quand le déphasage entre les signaux $\alpha(t)$ et $\alpha_{\infty}(t)$ est important, pour des valeurs de $U_{c}$ qui tendent vers zéro $\left(\left(W / U_{c}\right) \rightarrow \infty\right)$ pour lesquelles, par rapport à l'équation générale du profil de $C / C_{0}$, une suraccumulation de sédiments dans les couches de la colonne d'eau situées près du fond est provoquée par l'équation de transport 1DV régissant la dynamique verticale des matières en suspension, suraccumulation qui disparaît quand la valeur de $U_{c}$ remonte. Cet écart par rapport à l'équation générale du profil est en 
grande partie un biais des modélisations car dans ces conditions le dépôt doit se produire.

- Avec dépôt ou érosion, si les variables indépendantes du problème $\left(U_{c}, W\right.$ et $\left.d\right)$ restent constantes et si les échanges solides avec le fond sont proportionnels à la vitesse de chute des sédiments multipliée par la concentration au fond (paramètres $p$ et $q$ constants), les modélisations 1DV montrent que le profil du rapport $C / F\left(z^{\circ} ; \alpha\right)$ tend toujours vers une forme terminale qui reste similaire par la suite.

Si ces conditions persistent suffisamment longtemps, une forme terminale définie par $G\left(z^{\circ} ; \beta_{\infty}\right)$ est atteinte. On observe ainsi une stationnarité du paramètre $\beta_{\infty}$ si bien que les profils de $C$ peuvent être modélisés par une fonction $C_{R} \times F\left(z^{\circ} ; \alpha\right) \times G\left(z^{\circ} ; \beta_{\infty}\right)$, où $F\left(z^{\circ} ; \alpha\right)$ est la fonction décrivant le profil $C / C_{0}$ en régime non stationnaire sans dépôt et sans érosion. Si la stationnarité n'est pas atteinte pour $\beta$ le profil de $C$ n'est pas décrit exactement par $C_{R} \times F\left(z^{\circ} ; \alpha\right) \times G\left(z^{\circ} ; \beta\right)$, mais cette expression reste cependant une approche satisfaisante.

Les principaux résultats numériques de cette étude ont été obtenus avec un coefficient de diffusion turbulente $K_{z}$ invariant selon $z$. La même démarche peut être appliquée pour $K_{z}$ variant avec $z$, ce qui implique une éventuelle modification des fonctions $F\left(z^{\circ} ; \alpha\right)$ et $G\left(z^{\circ} ; \beta\right)$ (voir appendice 1$)$.

Pour $K_{z}$ constant sur la verticale, les fonctions utilisées sont $F\left(z^{\circ} ; \alpha\right)=\exp \left(-\alpha z^{\circ}\right)$ et $G\left(z^{\circ} ; \beta\right)=\exp \left(-\beta\left(1-z^{\circ}\right)^{2}\right)$. La valeur terminale du paramètre $\alpha$ est $\alpha_{\infty}=P^{e}$ et celle de $\beta$ est soit une fonction $\beta_{\infty}(p, \alpha)=0,5 p \alpha$, en cas de dépôt, soit une fonction $\beta_{\infty}(q, \alpha)$ en cas d'érosion (équation 35). Selon le modèle phénoménologique développé dans cet article, les variations de $\alpha$ et de $\beta$, sont simulées par les équations 40A et 40B respectivement. Dans ce modèle $\alpha$ et $\beta$ convergent uniformément sur toute la colonne d'eau vers $\alpha_{\infty}$ et $\beta_{\infty}$ respectivement, ce qui est une approximation du problème car la convergence réelle s'avère être plus rapide au fond et en surface que sur le reste de la colonne d'eau.

La meilleure validation du modèle phénoménologique est sa capacité avérée à reproduire la dynamique verticale des matières en suspension avec des résultats très proches de ceux issus d'une solution numérique des équations théoriques 1DV avec une discrétisation de la colonne d'eau qui utilise au moins 50 couches.

Dans les applications aux cas réels les erreurs liées au modèle phénoménologique sont acceptables si l'érosion et le dépôt sont bien paramétrés. Par contre, si pour des conditions de dépôt on impose $p=0$, les valeurs des erreurs relatives atteignent dans nos modélisations une valeur de 0,40 quand $\left(U_{c-\text { moy }} \times T / d\right) \approx 7$ et $P^{e}=12$ (voir figure $5 \mathrm{~b}$ ). Cette erreur est en grande partie un artefact des modélisations car ce cas est extrême, avec des conditions de mise en dépôt très marquées.

Dans la mesure où le modèle phénoménologique s'approche de la solution théorique du problème, les deux conclusions suivantes sur le comportement sédimentaire reflètent une réalité, au moins en moyenne sur la colonne d'eau : 
Description non stationnaire de la distribution verticale des sédiments transportés en suspension par les écoulements à surface libre, en présence de dépôt et d'érosion : 9.27

- Selon ce modèle la valeur de $\alpha$ tend toujours vers $P^{e}$. Sans érosion et sans dépôt, quand $\alpha \approx P^{e}$ le profil vertical de la concentration est dans des conditions proches de l'équilibre stationnaire sur toute la colonne d'eau. Si les variables indépendantes du problème $\left(U_{c}, W\right.$ et $\left.d\right)$ restent constantes, la durée nécessaire pour parvenir à cet équilibre peut être caractérisée par une constante de temps $\tau_{\alpha}=d /\left(c_{\alpha} \times U_{c}\right)$, si bien qu'après un temps $t=4,6 \times \tau_{\alpha}$ on aura : $\left(\alpha_{\infty}-\alpha\right)=\left(\alpha_{\infty}-\alpha_{\text {init }}\right) / 100$, où $\alpha_{\text {init }}$ désigne la valeur initiale de ce paramètre (à $t=0$ ).

Cette expression de la constante de temps se justifie par le fait que pour parvenir aux conditions d'équilibre les sédiments doivent se redistribuer sur toute la hauteur $d$ de la colonne d'eau et que la vitesse des transferts verticaux de mélange des sédiments est contrôlée par $U_{c}$.

- Le paramètre adimensionnel $\beta$ du modèle est en relation avec les effets des échanges solides avec le fond, sur le profil vertical de la concentration. Après une période de dépôt ou d'érosion, quand les échanges solides avec le fond cessent, la valeur de $\beta$ tend vers zéro au fur et à mesure que les sédiments se redistribuent dans la colonne d'eau, se rapprochant ainsi des conditions sédimentaires soit stationnaires, soit non stationnaires sans dépôt et sans érosion. Lorsque les variables indépendantes du problème sont constantes et que $p$ ou $q$ reste constant en cas de dépôt ou d'érosion respectivement, la durée nécessaire pour que $\beta$ parvienne à sa valeur terminale $\beta_{\infty}$ est caractérisée par une constante de temps $\tau_{\beta}=d /\left(c_{\beta} \times U_{c}\right)$.

On doit signaler que cette recherche s'est développée en vue des applications aux sédiments fins, mais les résultats s'appliquent à tous les matériaux transportés en suspension par les cours d'eau sous condition que les variables du problème soient bien paramétrées.

En conclusion finale, on peut affirmer que le modèle phénoménologique développé dans le cadre de cet article pour décrire le profil vertical de la concentration dans les modélisations hydrosédimentaires 2DH permet d'améliorer considérablement la précision et la qualité des informations qui peuvent être obtenues de ces modélisations.

\section{Appendices}

7.1 Appendice 1: Solutions analytiques pour le profil vertical de la concentration en matières en suspension dans un cours d'eau

Si le régime sédimentaire est stationnaire et uniforme (sans érosion et sans dépôt) l'intégration de l'équation 8 permet d'obtenir la loi de Rouse-Vanoni généralisée pour la distribution verticale des matières en suspension en régime stationnaire (ORTON \& KINEKE, 2001 ; SANCHEZ et al., 2005), à savoir :

$$
\left.C(z)\right|_{\text {stat }}=C_{0} \exp \left(-\int_{0}^{z} \frac{W}{K_{z}} \mathrm{~d} z\right)
$$


La solution de cette équation dépend des formulations retenues pour modéliser $W$ et $K_{z}$. Dans ce manuscrit on a retenu $W$ et $K_{z}$ constants pour obtenir l'équation 10 .

Une autre solution, due à ROUSE (1937), est obtenue en supposant $W$ constant et le coefficient de diffusion turbulente $K_{z}$ modélisé par :

$K_{z}=\kappa U_{c} z\left(1-z^{\circ}\right)$

La solution qui est valable pour $z \geq a$, est obtenu sachant que :

$\int_{a}^{z} \frac{\mathrm{d} z}{K_{z}}=\frac{1}{\kappa U_{c}} \ln \left[\frac{z(d-a)}{a(d-z)}\right]$

par conséquent on peut écrire :

$\left.C(z)\right|_{\text {stat }}=C_{a} \exp \left(-\frac{W}{\kappa U_{c}} \ln \left[\frac{z(d-a)}{a(d-z)}\right]\right)=C_{a}\left[\frac{a(d-z)}{z(d-a)}\right]^{W /\left(\kappa U_{c}\right)}$

où $C_{a}$ est la concentration de référence au niveau $z=a$ près du fond. L'évaluation du rapport $C_{a} / \bar{C}$ à partir de cette expression est très sensible à la valeur retenue pour $a$, laquelle selon certains auteurs, est égale à $0,05 \times d$, mais selon d'autres auteurs celle-ci est du même ordre de grandeur que la rugosité du fond.

D'une façon plus générale, si $K_{z}$ est une fonction de $z$ et si $W$ varie en fonction de la concentration locale $C$ suivant la loi de puissance qui peut être écrite comme suit :

$W=W_{0}\left(\frac{C}{C_{0}}\right)^{1 / r}$

où $W_{0}$ est la valeur de la vitesse de chute associée à la concentration au fond $C_{0}$, et $r$ une constante, alors, le profil vertical de la concentration est décrit par :

$\left.C(z)\right|_{\text {stat }}=C_{0}\left(\frac{\lambda_{0}}{\lambda_{z}}\right)^{r}$

avec $\lambda_{0}=r / W_{0}$, et $\lambda_{z}$ qui est donné par :

$\lambda_{z}=\lambda_{0}+\int_{0}^{z} \frac{\mathrm{d} z}{K_{z}}$

Ces trois dernières expressions restent valables, si d'un point de vue similaire à celui adopté pour classer le diamètre des grains de sable dans les études granulométriques, la vitesse de chute est distribuée selon une loi gamma de paramètres $r$ et $\lambda_{z}$ (SANCHEZ, 2006). 
Description non stationnaire de la distribution verticale des sédiments transportés en suspension par les écoulements à surface libre, en présence de dépôt et d'érosion : 9.29

7.2 Appendice 2: Relation entre la concentration au fond et la concentration moyenne sur la verticale en fonction des paramètres adimensionnels $\mathrm{du}$ modèle phénoménologique décrivant le profil vertical des matières en suspension D'après les équations 27 et 38 on peut écrire :

$$
\frac{C_{0}}{\bar{C}}=\frac{\exp (-\beta)}{\int_{0}^{1} \exp \left(-\alpha z^{\circ}\right) \exp \left(-\beta\left(1-z^{\circ}\right)^{2}\right) d z^{\circ}}
$$

Cette expression admet une solution analytique pour $\beta=0$ et peut être évaluée à l'aide des tables d'intégration de la loi normale de Gauss pour $\beta=0,5 \alpha$. Cependant, dans un cas général, cette équation doit être calculée par intégration numérique à l'aide de l'expression suivante :

$$
\frac{C_{0}}{\bar{C}}=\frac{\exp (-\beta)}{\sum_{i=1}^{N} \exp \left(-\alpha z^{\circ}{ }_{i}\right) \exp \left(-\beta\left(1-z^{\circ}{ }_{i}\right)^{2}\right) \Delta z^{\circ}}
$$

La figure 18 montre $C_{0} / \bar{C}$ en fonction de $\beta / \alpha$ pour quelques valeurs de $\alpha$.

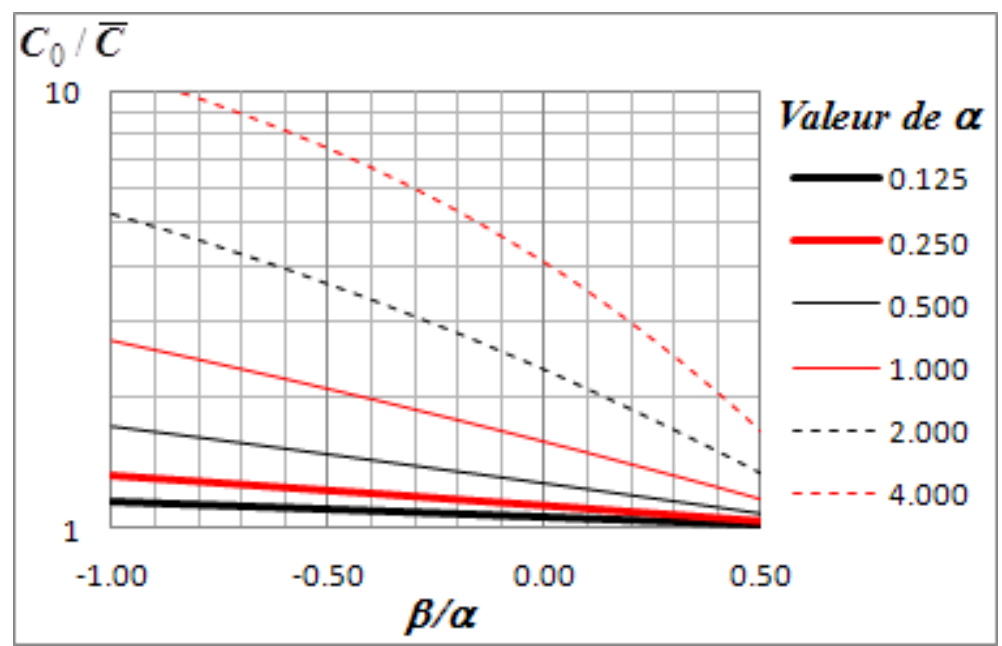

Figure 18. Variation de $C_{0} / \bar{C}$ en fonction de $\beta / \alpha$ pour quelques valeurs de $\alpha$ en conformité avec modèle phénoménologique décrivant le profil vertical des matières en suspension développé dans ce manuscrit.

\section{Références bibliographiques}

BELINSKY M., RUBIN H., AGNON Y., KIT E., ATKINSON J.F. (2005). Characteristics of resuspension, settling and diffusion of particulate matter in a water column. Environmental. Fluid Mechanics, Vol. 5, pp 415-441. doi:10.1007/s10652-004-7302-3 KRONE R.B. (1986). The significance of aggregate properties to transport processes. In A.J. Mehta (Ed.), Estuarine cohesive sediment dynamics, Coastal and estuarine studies n 14 (pp. 66-84). Springer, Berlin. doi:10.1007/978-1-4612-4936-8_4 
LUMBORG U., WINDELIN A. (2003). Hydrography and cohesive sediment modelling: application to Rømø Dyb tidal area. Journal of Marine Systems, Vol. 38, pp 287-303. doi:10.1016/S0924-7963(02)00247-6

MEHTA A.J. (1986). Characterization of cohesive sediment properties and transport processes in estuaries. In A.J. Mehta (Ed.), Estuarine cohesive sediment dynamics, Coastal and estuarine studies $n^{\circ} 14$ (pp. 290-325). Springer, Berlin.

doi:10.1007/978-1-4612-4936-8_15

ORTON P.M., KINEKE G.C. (2001). Comparing calculated and observed vertical suspended-sediment distributions from a Hudson River estuary turbidity maximum. Estuarine, Coastal and Shelf Science, Vol. 52, pp 401-410. doi:10.1006/ecss.2000.0747

ROUSE H. (1937). Modern conceptions of the mechanics of fluid turbulence. Transactions of the American Society of Civil Engineers, Vol. 102, pp 463-541.

SANCHEZ M. (2006). Settling velocity of the suspended sediment in three high-energy environments. Ocean Engineering, Vol. 33 (5-6), pp 665-678. doi:10.1016/j.oceaneng.2005.05.009

SANCHEZ M., GRIMIGNI P., DELANOË Y. (2005). Steady-state vertical distribution of cohesive sediments in a flow. Comptes Rendus Geoscience, Vol. 337, pp 357-365. doi:10.1016/j.crte.2004.10.020

TEETER A.M. (1986). Vertical transport in fine-grained suspension and newlydeposited sediment. In A.J. Mehta (Ed.), Estuarine cohesive sediment dynamics, Coastal and estuarine studies $n^{\circ} 14$ (pp. 170-191). Springer, Berlin. doi:10.1007/978-1-4612-4936-8_9

\section{Note complémentaire au manuscrit indexé DOI:10.5150/revue-paralia.2013.009}

Note soumise le 19 octobre 2015, acceptée le 6 novembre 2015, en ligne le 9 novembre 2015.

Des validations numériques complémentaires dont une partie a été publiée par SANCHEZ (2014), ont montré qu'en cas d'érosion, le paramètre $\beta_{\infty}$ peut être relié au produit $q \alpha$ par une expression unique qui est compatible avec la condition de frontière relative aux échanges de sédiments avec le fond :

$\beta_{\infty}=-0,50 q \alpha \quad$, pour toute valeur de $q \alpha \quad$ (Eq. 35 modifiée) En cas de dépôt $\left(D^{e f}>0\right)$ dans cet article le coefficient $c_{\beta}$ a été rattaché à $\beta$ par l'équation 32 , sur la base des résultats de simulations numériques effectuées avec $\beta=\beta_{\infty}$. Quand $\beta \neq \beta_{\infty}$ les résultats s'améliorent si le coefficient $c_{\beta}$ est rattaché à $\beta_{\infty}$ à travers l'équation 32 modifiée ci-dessous (voir SANCHEZ, 2014) :

$c_{\beta}=\left\{\begin{array}{lll}0,667+0,3 \times \beta_{\infty} & , s i: & D^{e f}>0 \\ 0,667 & , s i: & D^{e f}=0\end{array}\right.$

(Eq. 32 modifiée)

\section{Référence bibliographique :}

SANCHEZ M. (2014). Distribution non stationnaire des matières en suspension dans une couche limite oscillant à haute fréquence. XIII ${ }^{\mathrm{ème}}$ Journées Nationales Génie Côtier - Génie Civil, Dunkerque, pp 495-504. doi:10.5150/jngcgc.2014.054 